The Astrophysical JournaL, 466:623-637, 1996 August 1

(C) 1996. The American Astronomical Society. All rights reserved. Printed in U.S.A.

\title{
WEAK GRAVITATIONAL LENSING BY GALAXIES
}

\author{
Tereasa G. Brainerd, ${ }^{1,2}$ Roger D. Blandford, ${ }^{3}$ AND IAN SMAIL ${ }^{4,5}$ \\ Received 1995 March 20; accepted 1996 February 16
}

\begin{abstract}
We report a detection of weak, tangential distortion of the images of cosmologically distant, faint galaxies due to gravitational lensing by foreground galaxies. A mean image polarization of $(p)=$ $0.011 \pm 0.006$ ( $95 \%$ confidence bounds) is obtained for 3202 pairs of source $\left(23<r_{s} \leq 24\right)$ and lens $(20$ $\left(20 \leq r_{d} \leq 23\right)$ galaxies with projected separations of $5^{\prime \prime} \leq \theta \leq 34^{\prime \prime}$. Averaged over annuli of inner radius $5^{\prime \prime}$ and outer radius $\theta_{\max }$, the signal is string for lens-source separations of $\theta_{\max } \lesssim 90^{\prime \prime}$ consistent with quasi-isothermal galaxy halos extending to large radii $\left(\gtrsim 100 h^{-1} \mathrm{kpc}\right)$. The observed polarization is also consistent with the signal expected on the basis of simulations incorporating measured properties of local galaxies and modest extrapolations of the observed redshift distribution of faint galaxies (to which the results are somewhat sensitive). From the simulations we obtain formal best-fit model parameters for the dark halos of the lens galaxies that consist of a characteristic circular velocity of $V^{*} \sim 220 \pm 80 \mathrm{~km} \mathrm{~s}^{-1}$ and characteristic radial extent of $s^{*} \gtrsim 100 h^{-1} \mathrm{kpc}$. The predicted polarization based on the model is relatively insensitive to the characteristic radial extent of the halos, $s^{*}$, and very small halos $\left(s^{*} \sim 10 h^{-1}\right.$ $\mathrm{kpc}$ ) are excluded only at the $2 \sigma$ level. The formal best-fit halo parameters imply typical masses for the lens galaxies within a radius of $100 h^{-1} \mathrm{kpc}$ on the order of $1.0_{-0.5}^{+1.2} \times 10^{12} h^{-1} M_{\odot}(90 \%$ confidence bounds), in agreement with recent dynamical estimates of the masses of local spiral galaxies. This is particularly encouraging as the lensing and dynamical mass estimators rely on different sets of assumptions. Contamination of the gravitational lensing signal by a population of tidally distorted satellite galaxies can be ruled out with reasonable confidence. The prospects for corroborating and improving this measurement seem good, especially using deep HST archival data.
\end{abstract}

Subject headings: galaxies: fundamental parameters - galaxies: halos — gravitational lensing

\section{INTRODUCTION}

The notion that cosmologically distributed masses might cause weak but measurable changes to the shapes of distant galaxies has a long history. The most striking example of this "cosmological distortion effect" (Kristian \& Sachs $1966)$ is the distortion of distant galaxies into giant arcs caused by rich clusters of galaxies (see, e.g., Fort \& Mellier 1994). Attempts have also been made to measure the weak distortion of distant galaxy images by mass fluctuations associated with large-scale structure (see, e.g., Kristian 1967; Valdes, Tyson, \& Jarvis 1983; Mould et al. 1994, hereafter Paper I). These studies have progressed to the point where they can produce interesting limits on the largescale distribution of mass in the universe. The purpose of this paper, however, is to show how weak gravitational lensing can be used to study the mass distributions on much smaller scales, those associated with individual galaxies.

Galaxies constitute the fundamental building blocks of luminous structure in the universe, yet we are largely ignorant of such basic physical parameters as their typical masses and radial extents. Popular theories of galaxy formation predict that most bright galaxies should reside in

\footnotetext{
${ }^{1}$ Los Almos National Laboratory, Theoretical Astrophysics (T6), MS B288, Los Alamos, NM 87545.

${ }^{2}$ Current address: Boston University, Department of Astronomy, 725 Commonwealth Avenue, Boston, MA 01581

${ }^{3}$ California Institute of Technology, Theoretical Astrophysics 130-33, Pasadena, CA 91125.

${ }^{4}$ The Observatories of the Carnegie Institution of Washington, 813 Santa Barbara Street, Pasedena, CA 91101.

${ }^{5}$ Current address: University of Durham, Department of Physics, South Road, Durham, DH1 3LE, UK.
}

massive $\left(\gtrsim 10^{12} M_{\odot}\right)$ dark halos that extend far beyond their optical radii $\left(\gtrsim 100 h^{-1} \mathrm{kpc}\right)$. Direct observational evidence to test such theories is, however, scarce. While observations of the central parts of galaxies provide good mass estimates for these regions, the lack of information on the form and extent of the dark halos of individual galaxies limits our determination of their masses. Observations of local galaxies, most importantly our own (see, e.g., Fich \& Tremaine 1991), indicate that the majority of bright spiral galaxies have dark halos which extend isothermally out to at least $\sim 30 \mathrm{kpc}$. Studies using the dynamical properties of ensembles of faint companions to samples of bright spirals favor a continuation of the halo out of radii $\sim 100 h^{-1} \mathrm{kpc}$ (Zaritsky \& White 1994). On purely theoretical grounds, if we suppose the mass of the halos increases linearly with radius out until their density reaches the critical density, they will have outer radii $\sim(2 / 3)^{1 / 2}\left(V_{c} / H_{0}\right) \sim 1-2 h^{-1} \mathrm{Mpc}$, where $V_{c}$ is the circular velocity. Alternatively, the halos of most giant galaxies may be truncated at radii smaller than one-tenth of this value.

In this paper we report on a detection of distortion of the shapes of distant galaxy images due to weak gravitational lensing by individual foreground galaxies. Using the observed gravitational lensing signal, we will then estimate the masses of typical field galaxies. The advantages of the lensing approach to the determination of galaxy masses are twofold. First, it is capable of probing the halos of galaxies out to very large radii, $r \gtrsim 100 h^{-1} \mathrm{kpc}$, where few classical techniques are viable. Second, the lensing analysis is relatively unaffected by the dynamical properties of the possibly unvirialized outer regions of the halos. Lack of detailed information about the dynamics at large radii in galaxies is one of the central problems for the application of dynamical 
mass estimators. The dynamical and lensing mass estimates depend upon different model assumptions and a comparison of the results obtained from the two techniques is one of the few ways in which the validity of these assumptions can be tested.

The signal we seek is a distortion of the images of faint galaxies resulting in a weak preference toward the tangential alignment of faint galaxies around brighter galaxies. If the faint galaxies are gravitationally lensed by the brighter systems, the major axes of their images will tend to lie perpendicular to the radius vectors joining the faint galaxies to the lens centers (Fig. 1). The strength of this signal depends upon the distances of the lens and source galaxies, the mass of the lens, and the angular separation of the lens and source on the sky. With statistical information from direct spectroscopic studies (see, e.g., Lilly 1993; Tresse et al. 1993) on the redshift distributions of both the source and lens galaxy samples, we can therefore solve for the typical masses of the lensing galaxies.

The detection of galaxy-galaxy lensing was first attempted by Tyson et al. (1984) using scans of photographic plates (see also Webster 1983) from which they searched for an excess of background galaxy images tangentially elongated with respect to brighter, candidate lens galaxies. The galaxy sample available to Tyson et al. was very large, $\sim 47,000$ background galaxies with $22.5<J<23.5$ and $\sim 12,000$ lens galaxies with $19<J<21.5$. Faint image position angles were measured for over 28,000 galaxy pairs and no statistically significant difference from an isotropic distribution was seen for galaxy separations greater than

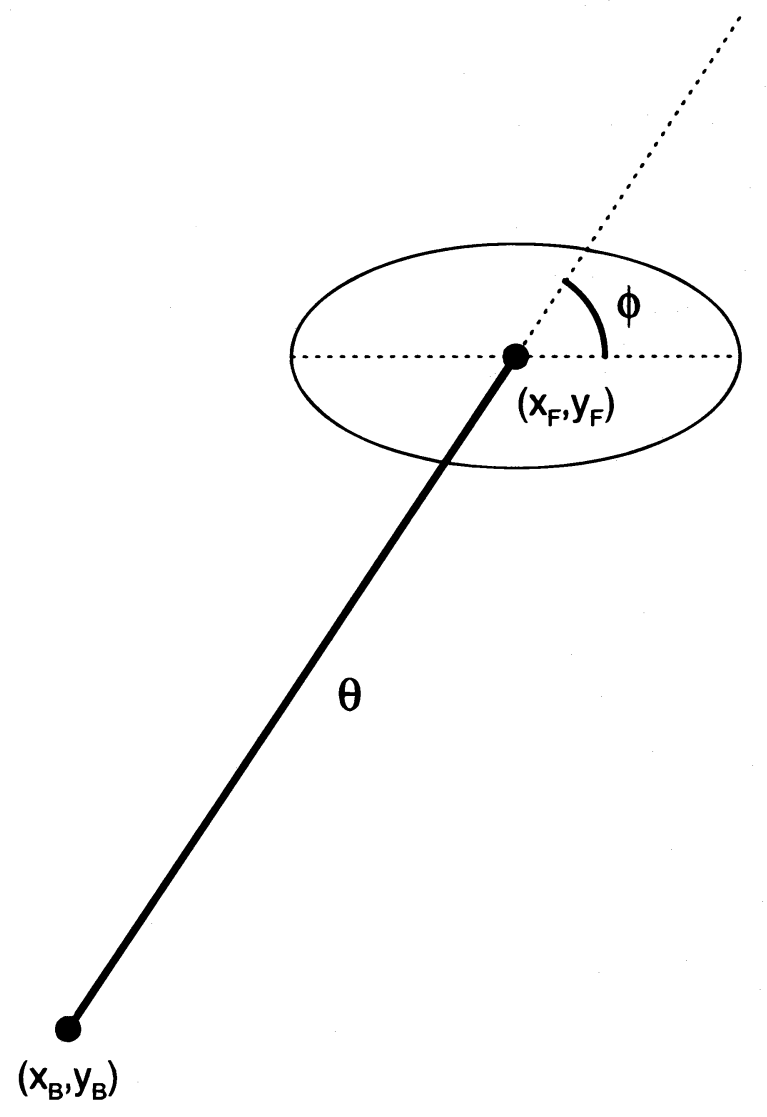

FIG. 1.-Orientation of faint galaxies relative to bright galaxies $\sim 3^{\prime \prime}$. Tyson et al. set a limit on galaxy-galaxy lensing for image separations $>5^{\prime \prime}$ and concluded that the typical galaxy circular velocity had a surprisingly small upper bound, $\$ 170 \mathrm{~km} \mathrm{~s}^{-1}$. Kovner \& Milgrom (1987) performed a careful calculation of the magnitude of the effect anticipated, taking into account integration over galaxy luminosity functions and distances as well as the correlation of internal, galaxian velocity dispersions with luminosity, and concluded that the observation of Tyson et al. was consistent with a circular velocity of $330 \mathrm{~km} \mathrm{~s}^{-1}$ in an untruncated halo.

In $\S 2$ we describe our observations and present our results for the detection of weak gravitational lensing of distant galaxy images by foreground galaxies, together with the analyses we have performed to attempt to understand the importance of systematic errors. In $\S 3$ we describe model calculations, both analytic and Monte Carlo, that we have used to translate our observed galaxy-galaxy lensing signal into a quantitive statement about galaxy masses and extents, the results of which are presented in $\S 4$. Finally, in $\S 5$ we relate our estimate of galaxy masses and radial extents to more conventional determinations of these quantities.

\section{MEASUREMENT OF GALAXY-GALAXY LENSING}

To determine roughly the expected strength of the galaxy-galaxy gravitational lensing signal, let us model a lens galaxy as a singular isothermal sphere with circular velocity $V_{c}$. An ellipticity $\sim 2 \pi V_{c}^{2} / c^{2} \theta$ will then be induced in the image of a background faint galaxy located an angular distance $\sim \theta$ from the lens. This is of the order of a few percent effect for galaxy pairs with separations $\theta \sim 30^{\prime \prime}$, where the lens galaxy is a typical bright spiral galaxy. In order to detect this signal in the presence of the noise associated with the intrinsic galaxy shapes, over a thousand foreground-background galaxy pairs must be measured. If a sufficiently large number of pairs are available, it may also be possible to use the dependency of the lensing signal on the distance from the lens center, $\theta$, to study the angular extent of galaxy halos. In the following sections we discuss an observational data set that should be of sufficent quality, depth, and size to allow a detection of galaxy-galaxy lensing.

\subsection{Observational Data}

The imaging data used in our analysis is of a single 9'.6 $\times 9^{\prime} .6$ blank field centered on $\alpha(1950)=17^{\mathrm{h}} 21^{\mathrm{m}} 07^{\mathrm{s}}$, $\delta(1950)=+49^{\circ} 52^{\prime} 21^{\prime \prime}$, taken in Gunn $r$. The data were acquired in periods of good seeing, $0.7-0.9$, using the direct imaging mode of the COSMIC imaging spectrograph (Dressler et al. 1996) on the $5 \mathrm{~m}$ Hale telescope, Palomar. These data have been used previously to study the coherent distortion of faint galaxy images due to weak gravitational lensing by large-scale structure (Paper I) and the angular clustering statistics of faint galaxies (Brainerd, Smail, \& Mould 1995). The reduction of the data to a catalog of detected objects is detailed in Paper I.

The final stacked $r$ frame used for our principal data analysis consists of a total of 19 individual frames with a cumulative exposure time of $24.0 \mathrm{ks}$. The final frame has a $1 \sigma$ surface brightness limit of $\mu_{r}=28.8 \mathrm{mag} \operatorname{arcsec}^{-2}$, seeing of $0.87 \mathrm{FWHM}$, and a total area of $90.1 \mathrm{arcmin}^{-2}$. The object catalog created from this frame using the 
FOCAS image analysis package (Valdes 1982) shows galaxy counts rising as $\log (d N / d r) \sim 0.31 r$ to the $\sim 97 \%$ completeness limit of $r=26.0$. Because of the presence of classical distortion in the corners of the frame, we restrict all analysis to only those objects that lie within a circle of radius 4.8 , centered on the chip. There are 4819 galaxies within the area brighter than $r=26.0$.

An additional, shallower $g$-band image of the same field is also available and is used here solely for the purpose of providing color information on the objects detected in the deep $r$ image. The $g$-band image has a total exposure time of $6.0 \mathrm{ks}$ and seeing of 1".2. The median color errors for galaxies detected on the $r$ image with $(g-r) \leq 1$ at $r \sim 26$ (approximately $80 \%$ of the total population) are $\Delta(g-r) \sim 0.2$.

In order to calculate the gravitational lensing signal yielded by our models and to estimate the lensing-induced mean image polarization of our faint objects, we shall require the distribution function of the intrinsic source galaxy ellipticities. To linear order, the source ellipticity distribution can be estimated from the ellipticity distribution of the images. We have, therefore, measured the distribution function of image ellipticities in our sample and find that it is adequately fitted by the normalized distribution function

$$
P_{\epsilon}(\epsilon)=64 \epsilon \exp [-8 \epsilon]
$$

with mean ellipticity $(\epsilon)=0.25 \pm 0.02$. In addition, the observed ellipticity distribution of the faint, partially resolved galaxies is in good agreement with that of the bright, well-resolved galaxies, and equation (2.1) above.

\subsection{Position Angle Probability Distribution}

In this section we investigate the orientation of faint galaxies relative to the directions to nearby bright galaxies (see Fig. 1). The unweighted second moments of the intensity were measured and a complex orientation, $\chi$, formed for each faint image. The modulus of $\chi$ is $\left(a^{2}-b^{2}\right) /\left(a^{2}+b^{2}\right)$, where $a^{2}$ and $b^{2}$ are the principal second moments of the intensity provided by FOCAS. (See Paper I for a discussion of alternative prescriptions for describing the image shapes.) For $a \sim b,|\chi|$ is approximately equal to the measured ellipticity, $\epsilon=1-b / a$, and this identification is adequate for our purposes. The phase of $\chi$ is twice the position angle, $\phi$, and we shall use the convention of measuring $\phi$ as the angle between the major axis of the equivalent ellipse and the radius vector, measured in a counterclockwise sense. We also combine positive and negative position angles so that $\phi$ is restricted to $[0, \pi / 2]$.

In the absence of distortion of the faint galaxy images, we expect a uniform distribution of their position angles for all projected separations, $\theta$, between the candidate lenses and sources. When the background galaxy images are gravitationally lensed by foreground galaxies, we expect a distribution of position angles that is nonuniform with a deficit of faint images oriented radially $(\phi=0)$ and an excess of faint images oriented tangentially $(\phi=\pi / 2)$. As we show in $\S 3$, the deviation of the distribution from uniform should exhibit a $\cos 2 \phi$ variation. At large projected separations, of course, the distribution of $\phi$ should become uniform.

In Figure 2 we show the observed faint galaxy orientation distribution, $P_{\phi}(\phi)$, for lens-source projected separations of $5^{\prime \prime} \leq \theta \leq 34^{\prime \prime}$. To determine $P_{\phi}(\phi)$, we use the 439 bright
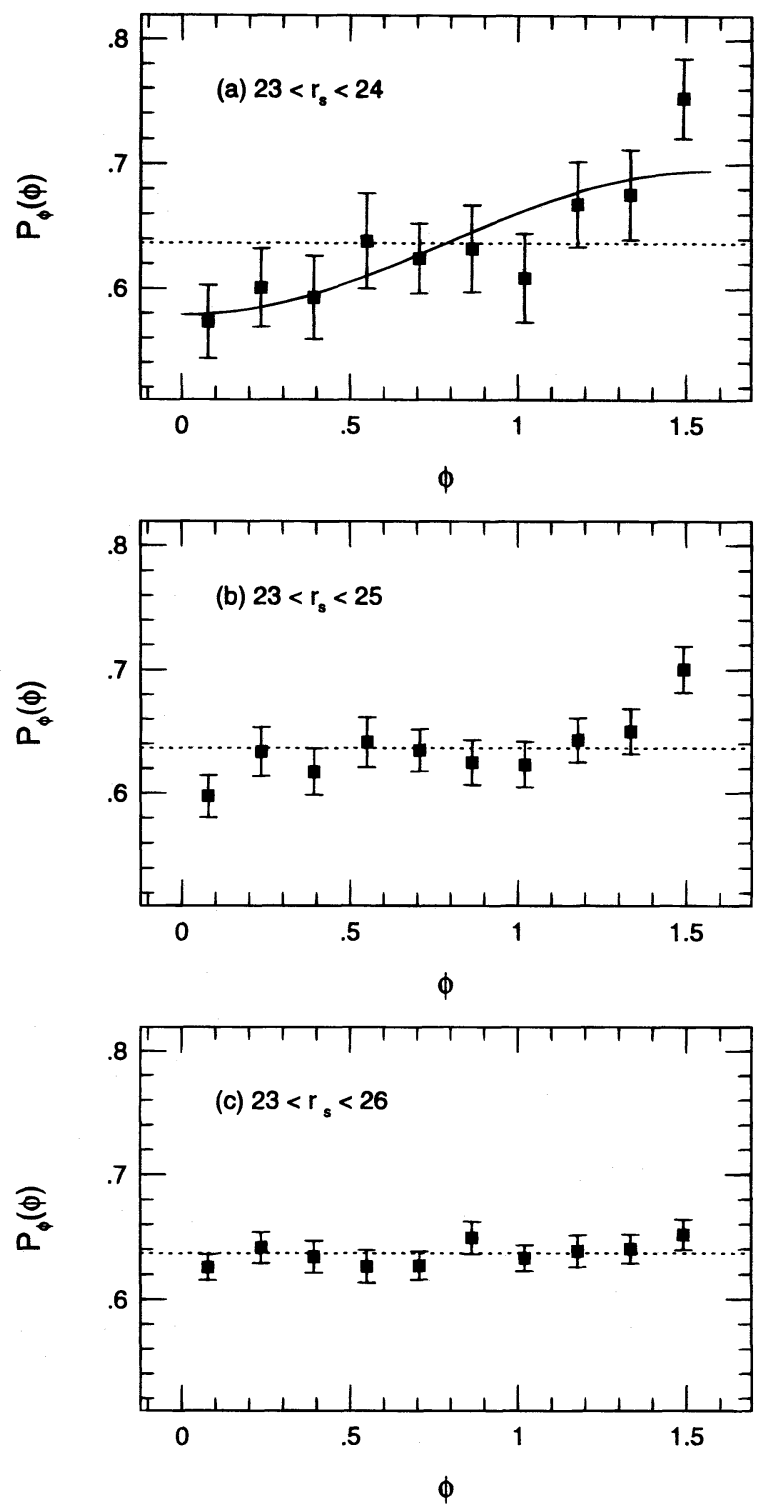

FIG. 2.-Probability distribution $P_{\phi}(\phi)$ of orientation of faint galaxies relative to the directions of bright galaxies with projected separations $5^{\prime \prime} \leq \theta \leq 34^{\prime \prime}$. The bright galaxies have $20 \leq r \leq 23$, and the faint galaxies have magnitudes in the ranges indicated in the text and figure panels. For the best case, $(a)$, of a nonuniform $P_{\phi}(\phi)$, the best-fitting theoretical $\cos 2 \phi$ variations is also shown.

galaxies with magnitudes in the range $20.0 \leq r_{d} \leq 23.0$ (the candidate lenses) and faint galaxies with magnitudes in the following ranges: (a) $23.0<r_{s} \leq 24.0$, (b) $23.0<r_{s} \leq 25.0$, and $(c) 23.0<r_{s} \leq 26.0$. The magnitude limits used to select the candidate lens galaxies are roughly equivalent to the depth of current faint spectroscopic surveys (see, eg., Lilly 1993; Tresse et al. 1993), which provide statistical redshift information on this population. The observed number of faint images, $N\left(r_{s}\right)$, and faint-bright pairs, $N\left(r_{s}, r_{d}\right)$, as a function of source limiting magnitude are summarized in Table 1.

The lower limit on $\theta$ was chosen so as to avoid overlapping faint and bright image isophotes. This is based upon visual inspection of the images of many of the candidate lenses and by noting that the mean half-light radius at $20 \leq r \leq 23$ is 0 ". 7 ; thus, the minimum annular radius corre- 
TABLE 1

Image Polarization and ReJection Confidence LeVels

\begin{tabular}{crcccc}
\hline \hline $\begin{array}{c}\text { Magnitude Range } \\
\text { (source galaxy } r \text {-magnitude) }\end{array}$ & $N\left(r_{s}\right)$ & $N\left(r_{s}, r_{d}\right)$ & $\begin{array}{c}\chi^{2} \text { Rejection } \\
\text { Confidence Level }\end{array}$ & $\begin{array}{c}\text { KS Rejection } \\
\text { Confidence Level }\end{array}$ & $\begin{array}{c}\langle p\rangle \\
(95 \% \text { Confidence Limits) }\end{array}$ \\
\hline $23.0<r_{s} \leq 23.5 \ldots \ldots \ldots \ldots \ldots$ & 207 & 1330 & $97.9 \%$ & $99.3 \%$ & $0.012 \pm 0.010$ \\
$23.0<r_{s} \leq 24.0 \ldots \ldots \ldots \ldots \ldots$ & 506 & 3202 & 98.6 & 99.9 & $0.011 \pm 0.006$ \\
$23.0<r_{s} \leq 24.5 \ldots \ldots \ldots \ldots \ldots$ & 1012 & 6323 & 98.6 & 99.6 & $0.006 \pm 0.004$ \\
$23.0<r_{s} \leq 25.0 \ldots \ldots \ldots \ldots \ldots$ & 1755 & 10870 & 97.3 & 99.2 & $0.005 \pm 0.003$ \\
$23.0<r_{s} \leq 25.5 \ldots \ldots \ldots \ldots \ldots$ & 2885 & 16734 & 16.1 & 21.5 & $0.000 \pm 0.003$ \\
$23.0<r_{s} \leq 26.0 \ldots \ldots \ldots \ldots \ldots$ & 4303 & 26412 & 19.4 & 60.0 & $0.001 \pm 0.002$ \\
\hline
\end{tabular}

sponds to $\sim 7$ scale lengths. The value of the outer radius of the annulus used here corresponds to an average impact parameter of $\sim 120 \mathrm{~h}^{-1} \mathrm{kpc}$ at our expected median lens redshift $\left(z_{m} \sim 0.5\right)$. It should be noted that, because of the size of the annulus, each candidate source is paired with a number of candidate lenses (see Table 1). For our set of candidate lenses and sources, this choice of annulus optimizes the signal to noise and it will be discussed in detail in $\S$ 3.6. The error bars on $P_{\phi}(\phi)$ are estimated from bootstrap resampling of the data.

From Figure 2, the (binned) distribution of faint image position angles appears nonuniform for the two brighter galaxy samples. In addition, $P_{\phi}(\phi)$ for the two brighter samples is qualitatively in agreement with what one would expect in the case of lensing by the bright foreground galaxies. The significance of the deviation of $P_{\phi}(\phi)$ from a uniform distribution is investigated using standard statistical techniques.

A $\chi^{2}$ test performed on the binned $P_{\phi}(\phi)$ in Figure 2 rejects a uniform distribution $\left[P_{\phi}(\phi)=2 / \pi\right]$ at a modest confidence level $(>97 \%)$ for both of the brighter samples and cannot reject a uniform distribution for the faintest sample. The sensitivity of the $\chi^{2}$ test to the arbitrary choice of bin size when using a continuous variable is well known. With this in mind, we consider the Kolmogorov-Smirnov (KS) test, which compares the observed continuous, cumulative distribution with a similar theoretical distribution, to be a better statistic for our purposes. KS tests performed on the cumulative $P_{\phi}(\phi)$ distributions corresponding to the binned distributions shown in Figure 2 reject uniform distributions at better than the $99 \%$ confidence level for both brighter source samples; however, we cannot rule out the hypothesis that our faintest sample is uniformly distributed. A summary of rejection confidence levels as a function of the source limiting magnitude is given in Table 1. Also shown in Table 1 are the corresponding rejection confidence levels for $P_{\phi}(\phi)$, where $23.0<r_{s} \leq 23.5,23.0<r_{s} \leq$ 24.5 , and $23.0<r_{s} \leq 25.5$. From this table it is clear that for $r_{s} \leq 25.0$, there is a strong rejection of the uniform distribution for $P_{\phi}(\phi)$, while for the deeper source samples $P_{\phi}(\phi)$ is consistent with a uniform distribution.

We do not view the absence of an obvious lensing signal in our faintest source sample as a strong concern. In a universe in which galaxies at fainter magnitudes are observed at progressively more distant epochs, it is predicted that the lensing signal will strengthen in fainter samples. Unfortunately, as we reach fainter in our universe, the typical angular size of galaxies decreases, and by $r \sim 26$ they have half-light radii of $\lesssim 00^{\prime \prime} 3$. This is small compared to our seeing and means that our faintest galaxies are only partially resolved, which leads to increasing errors in the determination of their ellipticities and position angles and degrades the gravitational lensing signal that we measure. Such a trend is clear from the data exhibited in Figure 2 and Table 1.

In determining $P_{\phi}(\phi)$, the largest contribution to the variance is the error in the determination of the position angles of the faint galaxies. For our purposes, the effect upon the faint images of seeing, sky noise, the detection alogrithm, etc, can be quantified in terms of the net error associated with the determination of the individual position angles. In the case of well-resolved images, of course, the error is a function of the image ellipticity. To investigate this, the imaging data were combined into two independent frames (one consisting of 10 frames; the other, of the remaining 9 frames), and objects on these two independent frames were found using the same detection process as was used for the full data set. The standard deviation in the faint object position angles as a function of $r_{s}$ and $\epsilon$ was then computed from a set of matched object catalogs. Results are summarized in Table 2, where it is apparent that the error in position angle becomes large for the faintest objects.

In our search for a statistically reliable gravitational lensing signal, then, we must settle for an optimal choice of limiting magnitude for the faint images, and this is $r \sim 24$ for our data. A source sample defined by $23.0<r_{s} \leq 24.0$ (Fig. $2 a$ ) is therefore adopted for our principal analysis.

As we anticipate $P_{\phi}(\phi)$ will exhibit a $\cos 2 \phi$ variation in the case that the faint images have indeed been gravitationally lensed by the brighter galaxies, we show in Figure $2 a$ the best-fitting $\cos 2 \phi$ variation for $P_{\phi}(\phi)$ for the subsample that will be used for our principal analysis.

\subsection{Possible Systematic Effects}

A number of tests were performed to investigate possible systematic effects in the data that would give rise to the observed nonuniform $P_{\phi}(\phi)$. For the following tests, lenssource pairs with projected separations of $5^{\prime \prime} \leq \theta \leq 34^{\prime \prime}$, lens galaxies with $20 \leq r_{d} \leq 23$, and source galaxies with $23<r_{s} \leq 24$ were considered.

TABLE 2

Standard Deviation in Faint Image Position ANGle

\begin{tabular}{cccc}
\hline \hline$\epsilon$ & $23<r_{s} \leq 24$ & $23<r_{s} \leq 25$ & $23<r_{s} \leq 26$ \\
\hline $0.0-0.1 \ldots \ldots$ & $35^{\circ}$ & $35^{\circ}$ & $35^{\circ}$ \\
$0.1-0.2 \ldots \ldots$. & 25 & 30 & 30 \\
$0.2-0.3 \ldots \ldots$. & 20 & 30 & 30 \\
$0.3-0.4 \ldots \ldots$. & 10 & 20 & 25 \\
$0.4-0.5 \ldots \ldots$ & 10 & 15 & 25 \\
$0.5-0.6 \ldots \ldots$ & 5 & 10 & 20 \\
$0.6-0.7 \ldots \ldots$ & 5 & 5 & 20 \\
\hline
\end{tabular}


To begin, a number of null tests were performed. From the data, $P_{\phi}(\phi)$ was computed for (1) $\phi$ taken to be the position angle of the lens galaxies relative to the lines connecting their centroids with those of the sources, (2) the position angle of the source images relative to lines connecting their centroids with random points (i.e., not corresponding to the centroids of the lenses), (3) the position angle of the source images relative to the lines connecting their centroids to bright stars on the frame, and (4) random position angles were substituted for the observed position angles of the faint images. For test (3), there are a total of 72 stars within the central 4.8 circle, which were selected using the same FOCAS parameters as the galaxies. In all four cases, the KS test indicates consistency with a uniform distribution (i.e., no "signal" is observed). The formal confidence levels at which the uniform distribution can be rejected are (1) $89.2 \%$, (2) $13.6 \%$, (3) $39.1 \%$, and (4) $26.0 \%$.

Additionally, the full data set was split into a number of subsamples and $P_{\phi}(\phi)$ computed for objects (1) with positive values of $\phi,(2)$ with negative values of $\phi$, (3) within a radius of 3.4 of the center of the chip, (4) at a radius greater than 3.4 of the center of the chip (but within the $4^{\prime} .8$ radius imposed for all data analysis), (5) within the north half-circle of radius $4.8,(6)$ within the south half-circle of radius 4.8 , (7) within the east half-circle of radius 4.8 , and (8) within the west half-circle of radius 4.8. In all cases, $P_{\phi}(\phi)$ is inconsistent with a uniform distribution. Rejection confidence levels are summarized in Table 3, where it is clear that, although the uniform distribution is rejected in each subsample, the rejection is not as strong as that for the full data set (see Table 1).

Next, we discuss the possible role of a point-spread function (psf) asymmetry in creating the observed signal. The presence of a noncircular psf or guiding errors introduces a preferred orientation in the object images and can give rise to a nonuniform $P_{\phi}(\phi)$ on very large angular scales. When the orientation of the faint galaxies relative to the bright galaxies is computed on small scales $(\lesssim 1 / 4$ the size of the frame), this effect is canceled out because of the fact that we can compute the orientations of the faint images in complete annuli around the majority of the bright centers. However, on scales $\gtrsim 1 / 2$ the size of the frame, the effect becomes very significant as we can no longer compute the orientations of the faint images in complete annuli around most of the centers. In general, such an edge effect at large scales gives rise to a nonuniform $P_{\phi}(\phi)$ that does not resemble that expected for a gravitational lensing signal, being of a larger amplitude and peaking at an angle $\phi$ corresponding

TABLE 3

Mean Polarization and Rejection ConfiDence LeVels for Subset OF Fiducial Data SET

\begin{tabular}{lcc}
\hline \hline Subsample & $\begin{array}{c}\text { KS Rejection } \\
\text { Confidence Level }\end{array}$ & $\langle p\rangle(95 \%$ Confidence Limits) \\
\hline $1 \ldots \ldots \ldots \ldots$ & $98.7 \%$ & $0.010 \pm 0.008$ \\
$2 \ldots \ldots \ldots \ldots$ & 99.0 & $0.013 \pm 0.008$ \\
$3 \ldots \ldots \ldots \ldots$. & 93.3 & $0.011 \pm 0.008$ \\
$4 \ldots \ldots \ldots \ldots$ & 96.2 & $0.011 \pm 0.009$ \\
$5 \ldots \ldots \ldots \ldots$ & 93.1 & $0.011 \pm 0.008$ \\
$6 \ldots \ldots \ldots \ldots$ & 99.1 & $0.011 \pm 0.008$ \\
$7 \ldots \ldots \ldots \ldots$ & 97.3 & $0.009 \pm 0.008$ \\
$8 \ldots \ldots \ldots \ldots$ & 97.2 & $0.013 \pm 0.009$ \\
\hline
\end{tabular}

to a combination of the preferred orientation of the faint images and their direction vectors relative to the bright centers.

In the case of our data, there is a slightly elliptical psf measured from the bright stars on the frame and corresponding weakly preferred image orientation. Computing $P_{\phi}(\phi)$ for our fiducial subsamples of galaxies, we find it is consistent with a uniform distribution on scales of $\sim 100^{\prime \prime}-$ $150^{\prime \prime}$ (as would be expected for a gravitational lensing signal), but on scales $\gtrsim 250^{\prime \prime}, P_{\phi}(\phi)$ is significantly nonuniform because of the edge effect. The form of $P_{\phi}(\phi)$ on these scales, unlike Figure 2, does not coincide with the expectations of a gravitational lensing signal at these scales, being of a larger amplitude and peaking at $\phi=\pi / 4$ with corresponding suppressions at $\phi=0$ and $\phi=\pi / 2$. Such a signal (both amplitude and shape) in our data is expected at this scale, given the observed preferred orientation of the objects (mean position angle of $\sim-5 \pi / 18$ ).

As a check for systematics associated with the image detection routines and second moment determinations, $P_{\phi}(\phi)$ was computed from a simulated data frame in which a signature of weak gravitational lensing was not included. Images on the simulated frame were assigned random locations and orientations. The galaxy parameters (ellipticities, scale sizes, and magnitudes) were chosen such that after the images were convolved with the telescope psf, the distribution of these parameters matched the observed distributions. The surface density of objects above the completeness limit was matched to that observed, and in addition, fainter galaxies were added by extrapolating the number counts $2 \mathrm{mag}$ fainter in order to simulate the effects of crowding and merging of these undetected faint galaxies on the detected objects. The pixellated images were convolved with the psf defined by the telescope and atmosphere, and sky noise was added to obtain the same detection limits as in the observed images. The simulated frame was then analyzed using FOCAS, and a catalog of objects was produced using the same procedure implemented for the actual data set. Using the parameters corresponding to Figure $2 a\left(5^{\prime \prime} \leq \theta \leq 34^{\prime \prime}, 20 \leq r_{d} \leq 23,23<r_{s} \leq 24\right)$, $P_{\phi}(\phi)$ for the simulated faint galaxy images was computed and found to be consistent with a uniform distribution (KS rejection confidence level of $7.3 \%$ ).

To test the effects of spillover of light from the bright lens galaxies, simulations were performed in which regions of radii 34" centered on each bright galaxy $\left(20 \leq r_{d} \leq 23\right)$ were selected from the data, and a simulated circular galaxy was randomly added into this region. The artificial galaxy was an $r=24$ exponential disk, which, after seeing convolution, had a scale size equal to the modal value for the observed $23<r_{s}<24$ sample (1"1 FWHM). FOCAS was then run on the subraster to locate the input galaxy and extract its centroid and second moments. This procedure was repeated 10 independent times for each of the 439 bright galaxies, and $P_{\phi}(\phi)$ for the artificial galaxies was computed in a number of annuli, centered on the bright galaxy. Taking the inner radius of the annulus to be $5^{\prime \prime}$ and increasing the outer radius incrementally from $10^{\prime \prime}$ to $30^{\prime \prime}$ in steps of $5^{\prime \prime}, P_{\phi}(\phi)$ was found to be consistent with a uniform distribution for all five annuli (KS rejection confidence levels of $25.5 \%$ $58.3 \%$ ). Independent annuli of radial width $5^{\prime \prime}$ centered on $7 " .5,12$. $5,17 " .5,22.5$, and 27.5 were used for calculations of $P_{\phi}(\phi)$ as a function of the differential separation of the observed bright galaxies and the simulated galaxies. Again, 
in all cases $P_{\phi}(\phi)$ was consistent with a uniform distribution (KS rejection confidence levels of $25.5 \%-77.6 \%$ ).

From the results of our various tests we therefore conclude that the signal observed in Figure 2 is real and not an artifact of the data set or errors in the analysis. In the following section, we develop a model to recreate the observed nonuniform $\mathrm{P}_{\phi}(\phi)$ using galaxy-galaxy gravitational lensing.

\section{SIMULATIONS OF GALAXY-INDUCED IMAGE DISTORTIONS}

In order to compare the magnitude of our apparent signal with theoretical expectation from gravitational lensing, we have carried out a variety of analytic and Monte Carlo simulations of weak lensing of galaxy images. To do this, we adopt a simple, fiducial galaxy model, compute the expected signal using analytic and Monte Carlo simulations, and then explore the sensitivity of the predictions to changes in our assumptions. In so doing, we are implicitly assuming that the majority of our lens galaxies form a oneparameter family as far as their gravitational properties outside $\sim 30 \mathrm{kpc}$ are concerned. Some cosmogonic theories posit that the outer parts of ellipticals are similar to the halos of spirals (see, e.g., de Zeeuw \& Franx 1991), a view that is supported by X-ray observations (see, e.g., Fabbiano 1989). We shall therefore not distinguish between the various morphological types present in our sample.

\subsection{Galaxy Mass Model}

In devising an appropriate galaxy model, two simplifications present themselves. First, we are quite unconcerned with the details of the galaxy centers as we expressly exclude $\theta \lesssim 5^{\prime \prime}$. We can therefore adopt a model galaxy potential that is singular as $r \rightarrow 0$. Second, almost all of our images are weakly distorted as they lie well outside the tangential critical curves formed by the galaxies. This implies that if the ellipticity in the potential is $\epsilon_{g}(r)$ at radius $r$, the average image polarization $\langle p\rangle\left(r, \epsilon_{g}\right)$ will differ from that of a circular lens with the same mass contained within a circle of radius $r$ by an amount $O\left[\epsilon_{g}^{2}\langle p\rangle(r, 0)\right]$. As $\epsilon \lesssim 0.3$ for the bulk of the images, this correction is small compared with the uncertainty implicit in the model. We therefore treat the galaxy potential as circularly symmetric. It should be emphasized at this stage that it is not at all likely that the outer parts of galaxies will have had time to relax completely into the ellipsoidal shapes associated with the luminous inner regions. In particular, the largest equipotentials associated with individual galaxies may be quite noncircular. However, as weak lensing depends linearly upon the mass distribution and we are only attempting a statistical measurement, our model corresponds to a circularly averaged mass distribution. Note that when an image is distorted by two or more distinguishable lens galaxies, their linear effects can be superposed.

After some experimentation, we have found that a simple model mass distribution for the dark matter halos that contains an outer characteristic scale, $s$, as a free parameter is

$$
\rho(r)=\frac{V_{c}^{2} s^{2}}{4 \pi \mathscr{G} r^{2}\left(r^{2}+s^{2}\right)},
$$

where $V_{c}$ is the deprojected circular velocity for $r \ll s$, and $\mathscr{G}$ is Newton's constant. For $r \gg s$, the density declines as $r^{-4}$, and the polarization signal becomes small.
The surface density associated with this space density is given by

$$
\begin{aligned}
\Sigma(R) & =\int_{-\infty}^{\infty} d x \rho\left[\left(R^{2}+x^{2}\right)^{1 / 2}\right] \\
& =\frac{V_{c}^{2}}{4 \mathscr{G} R}\left[1-\left(1+X^{-2}\right)^{-1 / 2}\right],
\end{aligned}
$$

where $X \equiv R / s$. The total mass is finite

$$
M=\frac{\pi s V_{c}^{2}}{2 \mathscr{G}},
$$

and the mass contained within a radius, $r$, is given by

$$
M(r)=\frac{V_{c}^{2} s}{\mathscr{G}} \tan ^{-1}(r / s) .
$$

We shall need the two-dimensional potential, relative to the potential at some large but finite radius, $R_{L}$ :

$$
\begin{aligned}
\Phi(R)= & -2 \int_{R}^{R_{L}} d R^{\prime} \frac{M\left(R^{\prime}\right)}{R^{\prime}} \\
= & \frac{-\pi V_{c}^{2} s}{\mathscr{G}}\left\{\left(1+X^{2}\right)^{1 / 2}-X\right. \\
& \left.-\ln \left[\left(1+X^{2}\right)^{1 / 2}+1\right]\right\},
\end{aligned}
$$

where we drop a constant, logarithmic contribution from the outer reference radius that does not contribute to the deflection and the polarization. In order to demonstrate that this potential is physically realizable, we compute an associated distribution function in Appendix A and demonstrate that it is stable to small perturbations.

\subsection{Image Polarization}

Using the model potential for an individual lens galaxy given by equation (3.1), we now compute the deflection angle of light rays and the resultant image polarization. Let the lens galaxy have angular diameter distance $D_{d}$ and the source galaxy have angular diameter distances $D_{s}, D_{d s}$ as seen from Earth and the lens, respectively. The deflection of a ray from the source is then given by

$$
\alpha(X)=\frac{2 D_{d s}}{D_{s} c^{2}} \frac{d \Phi}{d R}=\frac{2 \pi V_{c}^{2} D_{d s}}{D_{s} X c^{2}}\left[1+X-\left(1+X^{2}\right)^{1 / 2}\right]
$$

(see, e.g., Blandford \& Narayan 1992; Schneider, Ehlers, \& Falco 1993). Equating the image polarization, $p(X)$, to the induced ellipticity, we have

$$
p(\mathrm{X})=-\frac{D_{d} X}{s} \frac{d}{d X}\left[\frac{\alpha(X)}{X}\right]=p_{0} G(X),
$$

where a positive polarization corresponds to a net tangential elongation of the images. The coefficient $p_{0}$ is given by

$$
p_{0}=\frac{2 \pi V_{c}^{2} D_{d} D_{d s}}{s D_{s} c^{2}}=\frac{4 \mathscr{G} M D_{d} D_{d s}}{s^{2} D_{s} c^{2}},
$$

and the function $G(X)$ is

$$
G(X)=\frac{(2+X)\left(1+X^{2}\right)^{1 / 2}-\left(2+X^{2}\right)}{X^{2}\left(1+X^{2}\right)^{1 / 2}} .
$$

For $X \ll 1, G(X) \sim 1 / X$, whereas for $X \gg 1, G(X) \sim 2 / X^{2}$. Note also that $G(1)=0.88$ so that the polarization $p \sim p_{0}$ 


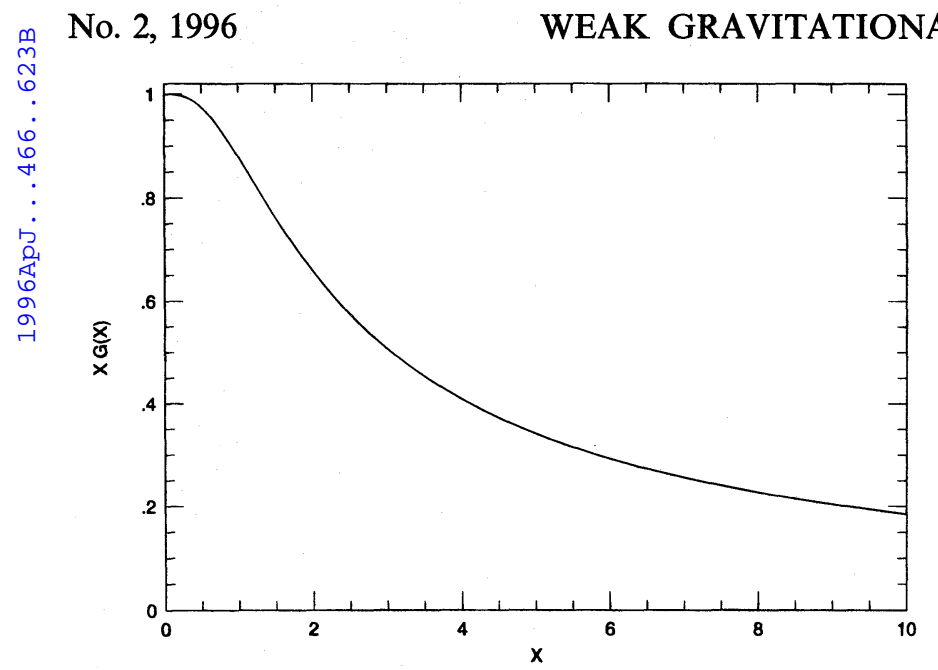

Fig. 3.-Scaled polarization variation with angle $\theta$ for a given galaxy. $G(X)$ is defined in eq. (3.9), and $X=R / s$.

when $R \sim s . G(X)$ is plotted Figure 3. Note that equations (3.6) and (3.7) allow us, in principle, to invert a measurement of $p(\theta)$ to obtain the average galaxy potential. However, our data are too sparse and our knowledge of the redshift distributions and the luminosity variation of galaxy properties is too incomplete to make this practical.

In order to use our model above to determine the expected image polarization for galaxies in an observational sample, we need to relate the luminosities of galaxies to the depths of their potential wells. To do this, we use the observations that the local spiral galaxy population can be well described by a luminosity function scaled to a characteristic luminosity $L^{*}$ (see, e.g., Loveday et al. 1992) and that these spirals appear to obey a Tully-Fisher relation that relates their luminosities, $L$, to their circular velocities, $V_{c}$ (see, e.g., Aaronson \& Mould 1983). With this in mind, we now introduce two scaling laws roughly consistent with these observations.

First, we assume that the circular velocity, $V_{c}$, scales as the fourth root of the total luminosity in a given band, in agreement with the Tully-Fisher relation (and also with the Faber-Jackson and fundamental plane laws for ellipticals if we treat $V_{c} / 2^{1 / 2}$ as the central velocity dispersion; see de Zeeuw \& Franx 1991). Introducing a scaling circular velocity, $V^{*}$, we have

$$
\frac{V_{c}}{V^{*}}=\left(\frac{L_{v}}{L_{v}^{*}}\right)_{r}^{1 / 4}
$$

where the subscript $r$-band and we assume that the emitted spectrum does not change with cosmological epoch. A fiducial estimate of $V^{*}$ is $220 \mathrm{~km} \mathrm{~s}^{-1}$ (see, e.g., Fich \& Tremaine 1991).

Our second scaling relation is more of a hypothesis. We assume that the total mass-to-light ratio of a galaxy is a constant independent of its luminosity. In other words, we suppose that there is a one-parameter family of galaxy potentials whose outer radii, $s$, scale as $s \propto M^{1 / 2} \propto L_{v}^{1 / 2} \propto$ $V_{c}^{2}$. Introducing a scaling radius, $s^{*}$, we have

$$
\frac{s}{s^{*}}=\left(\frac{L_{v}}{L_{v}^{*}}\right)_{r}^{1 / 2}=\left(\frac{M}{M^{*}}\right)^{1 / 2} .
$$

Imposing our galaxy scaling laws above, then, we conclude that $p_{0}$ does not depend explicitly upon the galaxy mass, and we can therefore write

$$
p_{0}=p^{*} \frac{D_{d s} D_{d} H_{0}}{c D_{s}},
$$

where

$$
p^{*}=0.10\left(\frac{V^{*}}{220 \mathrm{~km} \mathrm{~s}^{-1}}\right)^{2}\left(\frac{s^{*} h}{100 \mathrm{kpc}}\right)^{-1}
$$

is a reference polarization. The total mass of an $L^{*}$ galaxy in this model is

$$
M^{*}=1.8 \times 10^{12}\left(\frac{V^{*}}{220 \mathrm{~km} \mathrm{~s}^{-1}}\right)^{2}\left(\frac{s^{*}}{100 h^{-1} \mathrm{kpc}}\right) M_{\odot} .
$$

We now compute the expected mean image polarization from an observational investigation such as the one that we have described in $\S 2$. For simplicity, in our fiducial model we adopt an Einstein-de Sitter universe with angular diameter distances

$$
\begin{gathered}
D_{d}=\frac{2 c}{H_{0}} a_{d}\left(1-a_{d}^{1 / 2}\right), \quad D_{s}=\frac{2 c}{H_{0}} a_{s}\left(1-a_{s}^{1 / 2}\right), \\
D_{d s}=\frac{2 c}{H_{0}} a_{s}\left(a_{d}^{1 / 2}-a_{s}^{1 / 2}\right),
\end{gathered}
$$

and expansion factor $a_{d, \mathrm{~s}}=\left(1+z_{d, s}\right)^{-1}$.

We must also allow for a spectral (or " $K$-") correction, which can be fairly large in the $r$-band since the spectrum is quite steep at wavelengths blueward of this band (i.e., $\sim 6500 \AA$ ) in intermediate-redshift spirals. As we believe our lenses have redshifts $\sim 0.2-0.8$ (see below), we are concerned with spectral shapes in the range 3500-5500 $\AA$. Comparing with color measurements of faint galaxies, we find that $\alpha \equiv-d \ln L_{v} / d \ln v \sim 3$, very approximately. For ellipticals, the effective value of the spectral index, $\alpha$, is closer to 5 , and so they should be somewhat rarer at $z \sim 0.5$ in our $r$ selected faint galaxy sample than in an equivalently deep catalog selected in a redder passband. We also ignore the large dispersion in the colors of observed galaxies and treat them as a one-parameter family. We can then relate the apparent magnitude to the luminosity adopting $-18.5+5$ $\log h$ as the absolute $r$ magnitude of an $L^{*}$ galaxy. With this we obtain

$$
\frac{L_{v}}{L_{v}^{*}}=\left(\frac{H_{0} D_{d}}{c}\right)^{2}(1+z)^{3+\alpha} 10^{0.4(23.9-r)}
$$

at a given emitted frequency.

In order to proceed further, we need redshift distribution functions for the source and lens galaxies as a function of apparent magnitude. Lilly (1993) and Tresse et al. (1993) have presented $I$-selected redshift surveys that show that galaxies in a magnitude range comparable to that under consideration here $(I \sim 22$ or $r \sim 23)$ have a broad redshift distribution extending out to $z \sim 1$. As the results from our simulation are sensitive to the form of the redshift distribution used, we adopt a parameterized and normalized redshift distribution

$$
F(z, r)=\frac{\beta z^{2} e^{-\left(z / z_{0}\right)^{\beta}}}{\Gamma(3 / \beta) z_{0}^{3}} .
$$


For our fiducial model, we adopt $\beta=1.5$. The mode is $1.2 z_{0}$, the median is $z_{m}=1.4 z_{0}$, and the mean is $1.5 z_{0}$. More generally, we write

$$
z_{0}=k_{z}\left[z_{m}+z_{m}^{\prime}(r-22)\right] \quad 20<r<24,
$$

where the constant $k_{z}=0.7$ for $\beta=1.5$, and the derivative of the median redshift with respect to $r$ magnitude is $z_{m}^{\prime}=$ 0.1 fiducially. (For $20 \leq r \leq 23$, the parameterized redshift distribution is in good agreement with observation and the no-evolution model. For simplicity, we have assumed that the general form of the parameterization can be extended to $r=24$.)

Next, source galaxies with apparent magnitude $r_{s}$ are selected within an annular ring of other radius $\theta_{\max }$ and inner radius $f \theta_{\max }$. We now average the function $G(X)$ over this ring for lenses of fixed redshift $z_{d}$ and magnitudes $r_{d}$, obtaining

$$
\begin{aligned}
\bar{G}\left(z_{d}, r_{d}\right)= & \frac{\int_{D_{d} f \theta_{\max } / s}^{D_{d} \theta_{\max } / s} d X X G(X)}{\int_{D_{d} f \theta_{\max } / s}^{D_{\max }} d X X} \\
= & \frac{2 S}{\left(1-f^{2}\right)}\left\{(1-f)-\left(1+S^{2}\right)^{1 / 2}+\left(f^{2}+S^{2}\right)^{1 / 2}\right. \\
& \left.+2 S \ln \left[\frac{S+\left(1+S^{2}\right)^{1 / 2}}{S+\left(f^{2}+S^{2}\right)^{1 / 2}}\right]\right\}
\end{aligned}
$$

where $S=s / D_{d} \theta_{\max }$. For $S \ll 1, \bar{G} \propto S^{2}$; for $S \gg 1$, the relation becomes asymptotically, $\bar{G} \sim 1.7 S$.

We next integrate over the source redshift for a fixed lens:

$$
\langle p\rangle\left(z_{d}, r_{d}, r_{s}\right)=\left(\frac{p^{*} H_{0}}{c}\right) \bar{G}\left(z_{d}, r_{d}\right) D_{d} \int_{z_{d}}^{\infty} d z_{s} F\left(z_{s}, r_{s}\right)\left(\frac{D_{d s}}{D_{s}}\right) .
$$

Finally, we integrate over the redshift distribution of lens galaxies of magnitude $r_{d}$ to obtain

$$
\begin{aligned}
\langle p\rangle\left(r_{d}, r_{s}\right)= & \left.\frac{p^{*} H_{0}}{c}\right) \int_{0}^{\infty} d z_{d} \bar{G}\left(z_{d}, r_{d}\right) D_{d}\left(z_{d}\right) F\left(z_{d}, r_{d}\right) \\
& \times \int_{z_{d}}^{\infty} d z_{s} F\left(z_{s}, r_{s}\right)\left(\frac{D_{d s}}{D_{s}}\right) .
\end{aligned}
$$

Note that the polarization is directly proportional to the assumed velocity dispersion of an $L^{*}$ galaxy but depends nonlinearly on a single galaxy structural parameter, $s^{*}$.

\subsection{Fiducal Lens Model}

To summarize, our fiducial lens model in an Einstein-de Sitter universe uses

$$
\begin{gathered}
V^{*}=220 \mathrm{~km} \mathrm{~s}^{-1}, \quad s^{*}=100 h^{-1} \mathrm{kpc}, \quad \alpha=3, \\
\theta_{\max }=34^{\prime \prime}, \quad f=0.15, \quad \beta=1.5, \\
k_{z}=0.7, \quad z_{m}=0.47, \quad z_{m}^{\prime}=0.1 .
\end{gathered}
$$

In this model, the median redshift for an $r=22$ galaxy is $z=0.47$, in rough agreement with observations. At this redshift, $\theta_{\max }$ corresponds to $118 h^{-1} \mathrm{kpc}$. Using this model, then, we have evaluated equation (3.21) and show the results as a contour plot of $\langle p\rangle$ as a function of $r_{d}, r_{s}$ in Figure 4.

The image polarization measured from an observational sample is, of course, an average over a distribution of source magnitudes, $r_{s}$, and lens magnitudes, $r_{d}$. Assuming the

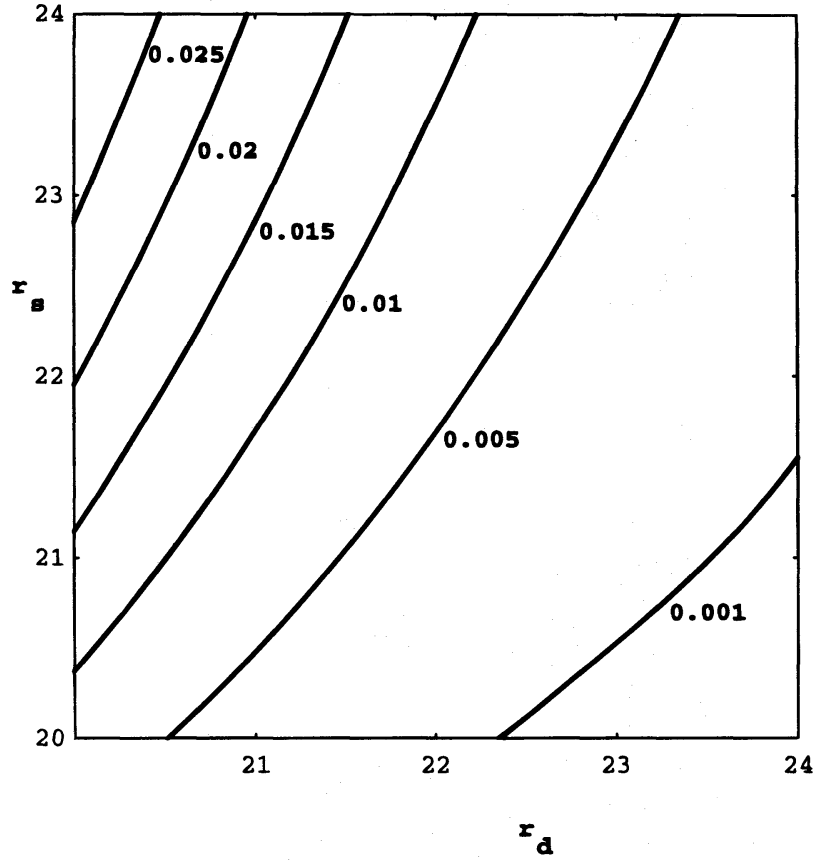

FIG. 4.-Theoretical variation of polarization as a function of source magnitude $r_{s}$ and lens galaxy magnitude $r_{d}$ for the fiducial $L^{*}$ galaxy model according to eq. (3.21).

number counts of galaxies as a function of $r$-magnitude follow a relation of the form $d N / d r=A 10^{0.31 r}$, we find the mean image polarization based upon our analytic model to be $\langle p\rangle=0.011$ for lenses and sources corresponding to our principal observational sample $\left(20 \leq r_{d} \leq 23\right.$ and $\left.23<r_{s} \leq 24\right)$.

\subsection{Calculation of Polarization}

Using linear theory, we can predict the distribution in image polarization. As in $\S 2.1$, we write the complex orientation of the image of a source galaxy as $\chi=\epsilon e^{2 i \phi}$. If the polarization is $p$, then to linear order, the intrinsic source orientation is $\chi_{0}=\chi-p$, and the effect of the lens galaxy is a simple translation on the $\chi$ plane. Let this translation be in the $x$-direction so that the normalized, observed distribution of orientations is

$$
f_{x}\left(\chi_{z}, \chi_{y}\right)=f_{x 0}\left(\chi_{z}-p, \chi_{y}\right),
$$

where $f_{x 0}$ is the intrinsic distribution in $\chi_{0}$. An unbiased estimator of the polarization is then given using

$$
\begin{aligned}
\langle\chi\rangle= & \int d \chi_{x} d \chi_{y} f_{x}\left(\chi_{x}, \chi_{y}\right) \chi_{x} \\
= & \int d \chi_{0 x} d \chi_{0 y} f_{x 0}\left(\chi_{0 x}, \chi_{0 y}\right) \chi_{0 x} \\
& +p \int d \chi_{x} d \chi_{y} f_{x}\left(\chi_{x}, \chi_{y}\right) \\
= & p \int d \chi_{x} d \chi_{y} f_{x}\left(\chi_{x}, \chi_{y}\right),
\end{aligned}
$$

where we have assumed that $f_{x 0}$ is isotropic. It is cleanest to estimate $p$ directly using

$$
p=\frac{\int d \chi_{x} d \chi_{y} f_{\chi}\left(\chi_{x}, \chi_{y}\right) \chi_{x}}{\int d \chi_{x} d \chi_{y} f_{\chi}\left(\chi_{x}, \chi_{y}\right)}
$$


However, what we actually measure is the distribution in $P_{\phi}(\phi)$ integrated over ellipticity. We therefore write

$$
\begin{aligned}
P_{\phi}(\phi) & =\int d \epsilon \epsilon f_{\chi 0}+p \cos 2 \phi \int d \epsilon \epsilon \frac{d f_{\chi 0}}{d \epsilon} \\
& =\left(\frac{2}{\pi}\right)\left[1-\langle p\rangle \cos 2 \phi\left\langle\epsilon^{-1}\right\rangle\right],
\end{aligned}
$$

where the best-fit ellipticity distribution, equation (2.1), yields $\left\langle\epsilon^{-1}\right\rangle=8.0$ and $\langle p\rangle$, the average polarization in the sample, should vary with the lens and source galaxy selection criteria.

\subsection{Monte Carlo Simulations}

To determine the best-fit halo parameters for our lensing model, we have constructed Monte Carlo simulations of gravitational lensing of background galaxies by foreground galaxies. For every observed object with $20 \leq r \leq 24$, we assign a galaxy to a random location on the Monte Carlo CCD frame (the angular clustering of the observed objects is weak [see, e.g., Brainerd et al. 1995] and has a negligible effect on the predicted signal). The galaxies are then assigned $r$-magnitudes and ellipticities drawn at random from the corresponding observed distributions and position angles uniformly distributed on $[-\pi / 2, \pi / 2]$. Based on its assigned $r$-magnitude, each galaxy is then given a redshift chosen from the parameterized redshift distribution given by equation (3.17), where the parameters assumed for the redshift distribution are those summarized by equation (3.22).

The mass distribution of the lens galaxies is modeled according to equation (3.1), and we assume all lenses follow same scaling relations with $V^{*}$ and $s^{*}$ (i.e., eq. [3.10] and eq. [3.11]), which are the parameters we wish to investigate. For each source galaxy, we compute the net image polarization induced by all foreground lens galaxies. Since we are dealing with the weak lensing limit, it is sufficient to compute the individual image polarizations due to distinguishable lenses and superpose them to obtain the net polarization. For the adopted galaxy redshift distributions, we find that roughly one third of the sources are lensed by only a single foreground galaxy, another third are affected by two lenses, and the remaining third encounter three or more significant deflectors.

Having computed the net polarization of each of the faint galaxy images, we then compute $\boldsymbol{P}_{\phi}(\phi)$ for the Monte Carlo images in exactly the same manner as the actual data shown in Figure $2 a$. By performing many $(\sim 1000)$ Monte Carlo realizations for a given pair of scaling parameters $\left(V^{*}, s^{*}\right)$, we determine a good estimate of the faint galaxy polarization for a particular lensing model. By comparing the predicted and observed $\langle p\rangle$ and using a $\chi^{2}$ minimization, we can then obtain formal best-fit parameters for the dark halos of the lenses. Results of this procedure and a discussion of the effects of realistic errors in the determination of faint object position angles on the predicted model image polarization are included in $\S 4$.

\subsection{Sensitivity to Model Parameters}

As mentioned above, the predicted image polarization is simply proportional to the square of the assumed fiducial circular velocity $V^{*}$. However, the dependence upon the outer radial scale, $s^{*}$, is more subtle. If we increase $s^{*}$ from its fiducial value to $\sim 300 h^{-1} \mathrm{kpc}$, keeping all other param- eters in the standard model (eq. [3.22)] the same, the polarization remains constant at 0.011 . Only if we reduce $s^{*}$ substantially is there any measurable reduction in the polarization. For example, changing $s^{*}$ to $30 h^{-1} \mathrm{kpc}$ reduces $\langle p\rangle$ from 0.011 to 0.009 . The reason for this behavior is that, within our standard model, most of the signal is contributed by lenses that are sufficiently close to the source on the sky that the line of sight passes through the isothermal part of the dark halo and, as $p^{*} \propto s^{-1}$ for a given velocity dispersion and $\bar{G} \propto s$, for large halos, the average polarization is approximately independent of $s$. The overall mass-to-light ratio will, however, increase in proportion to $s$.

Next, we vary the spectral index, $\alpha$, independently of the other parameters. Changing $\alpha$ from 3 to 2 reduces $\langle p\rangle$ from 0.011 to 0.009 . This comparatively weak variation indicates that our simplistic model for the $K$-correction is not a serious concern.

We now determine the sensitivity of the model predictions to the lens and source redshift distributions. Let us keep the median redshift (and its $z$ variation) constant but change the shape of the distribution. Reducing the high- $z$ tail by increasing $\beta$ from 1.5 to 2 (a Gaussian) results in a reduction of the mean polarization from 0.011 to 0.008 . Alternatively, we can keep the shape of the redshift distribution unchanged and change the median redshift. In this case, we find that increasing $z_{m}$ from 0.47 to 0.7 results in a mean polarization of 0.015 . Reducing $z_{m}$ to 0.3 results in a mean polarization of 0.009 . It turns out that our choice of variation of median redshift for the source galaxy distribution with magnitude more or less maximizes the predicted polarization and there is only a small sensitivity to $z_{m}^{\prime}$.

Similarly, there is little sensitivity to the world model. For example, changing from an Einstein-de Sitter universe to an empty universe $\left[\Omega_{0}=0, D_{d s}=c\left(2 H_{0} a_{d}\right)^{-1}\left(2+a_{s}+a_{d}\right)\left(a_{d}\right.\right.$ $\left.-a_{s}\right)$ ] results in a mean polarization of 0.012 .

Thus far, our measurement of the mean image polarization has used a fixed aperture around each lens galaxy of 34 , and each source galaxy is, therefore, paired with a number of lens galaxies in the computation of $P_{\phi}(\phi)$. While it is true that this results in a large number of faint-bright galaxy pairs, the statistical significance of the signal is not due to the number of pairs but, rather, to our choice of annulus being optimal for the detection of the signal from extended galaxy halos. It is clear from the Monte Carlo simulations that the majority of sources will have undergone at least two or more important deflections (see $\S 3.5$ ) and that in order to optimize one's ability to detect the signature of gravitational lensing, it is necessary to average the faint image orientation over all the possible deflectors. This is distinct from the approach in which the orientation of the faint image relative to only its nearest neighbor bright galaxy is investigated. If all the sources and all the lenses resided in single, disjoint planes in redshift and galaxies were point masses, the latter approach to the detection of the signal would be justified. In reality, however, the lenses and sources are distributed in redshift, and galaxies are not point masses. Thus, the nearest neighbor bright galaxy is (1) not the only possible lens and (2) not necessarily the strongest lens since many of the multiple deflections are of comparable magnitude. Therefore, averaging the faint image orientation over several near neighbor bright galaxies increases the signal-to-noise ratio, and in our case the choice of outer annulus of order 34" maximizes the signalto-noise ratio. 
To understand this, first observe that the induced polarization is linear in the mass distribution, and so it does not matter if several lens galaxies contribute to the net image polarization as long as they can be treated as randomly oriented relative to the sources. If we increase $\theta_{\max }$, the number of lens-source pairs increases as $\theta_{\max }^{2}$, and the random error diminishes as $\theta_{\max }^{-1}$. The polarization signal from these extra background galaxies diminishes as $\theta_{\max }^{-1}$ as long as we are still looking through the dark halos, with $\theta_{\max } \lesssim \theta_{c}$, and thus the signal-to-noise ratio ought to improve logarithmically with increasing $\theta_{\max }$. However, when the impact parameters exceed the lens outer radii, $s$, the signal-to-noise ratio deteriorates. Consequently, there is little gain in signal-to-noise ratio possible from increasing the aperture size beyond $s$. For example, increasing $\theta_{\max }$ from $34^{\prime \prime}$ to $60^{\prime \prime}$ leads to a reduction in the mean polarization signal $\langle p\rangle$ from 0.011 to 0.006 , a reduction by a factor 1.8 , even though the number of galaxy pairs increases by a factor 3.1 , which should reduce the noise by a factor 0.57 , leaving the signal-to-noise ratio constant. Conversely, reducing $\theta_{\max }$ to $15^{\prime \prime}$ increases $\langle p\rangle$ by a factor of 2.2 but reduces the number of pairs by 0.18 so that the signal-tonoise ratio diminishes by 0.8 .

\section{RESULTS}

Using the functional form for $P_{\phi}(\phi)$ given by equation (3.26) and fitting to the observations, we obtain $\langle p\rangle=0.011 \pm 0.006$ (95\% confidence bounds) for our fiducial galaxy sample $\left(20.0 \leq r_{d} \leq 23.0,23.0<r_{s} \leq 24.0\right)$ and $5^{\prime \prime} \leq \theta \leq 34^{\prime \prime}$. The confidence limits are the formal bounds obtained from a weighted least-squares fit of equations (3.26) to the data in Figure 2a. As consistency check on the mean polarization obtained for the entire fiducial galaxy sample, we have also determined $\langle p\rangle$ for the eight subsamples of the data described in $\S 2.3$. The mean polarizations obtained for the subsamples agree well with each other and with $\langle p\rangle$ obtained using the entire data set (see Table 3).

As discussed in $\S 2$, we cannot create such a signal from systematic effects in either our data set or our analysis. We therefore accept that the signal is real and search for an astrophysical origin. In this regard, two processes present themselves as obvious contenders to produce a preferential alignment of faint galaxy images around brighter systems: tidally distorted companions or gravitational lensing.

We now show that companion galaxies are unlikely to be the source of the observed signal. Limits can be set on the magnitude of the contamination due to tidally induced tangential elongations of genuine satellite galaxies of the lens galaxies (see Phillips 1985; Tyson 1985) from the clustering strength of the galaxies. Fortunately, the clustering statistics of the galaxies in this field have already been determined (Brainerd et al. 1995). We estimate the angular cross correlation function for galaxies in our primary data set with $20 \leq r_{d} \leq 23,23<r_{s} \leq 24$ to be

$$
w(\theta)=0.6\left(\frac{\theta}{1^{\prime \prime}}\right)^{-0.9},
$$

where we have computed the integral constraint directly for our frame and allowed a $15 \%$ stellar contamination of the object catalog (see Brainerd et al. 1995). Let us suppose that the mean polarization associated with intrinsic tidal effects is $p_{t}$. Our cursory examination of images of nearby galaxies reveals that $\left|p_{t}\right|<0.1$ for satellites within a magnitude fainter of their neighbour and separations $\sim 100 \mathrm{kpc}$. The contribution of the tidal elongation to the observed polarization signal should therefore be of order $1.6 w\left(\theta_{\max }\right) p_{t}$, which is smaller in modulus than 0.004 for $\theta_{\max }=34^{\prime \prime}$ and of order $2.3 \sigma$ below our measured image polarization. We therefore conclude that a contribution to the observed polarization due to a population of tidally distorted dwarfs can be ruled out with moderate confidence. The converse calculation that illustrates the effect of gravitational lensing on the measured autocorrelation function of galaxies at faint magnitudes is given in Appendix B.

We conclude that the most probable cause of the signal reported in $\S 2$ is the distortion of the distant galaxy images due to gravitational lensing by foreground galaxies. Our fiducial model, equation (3.22), yields a prediction of $\langle p\rangle=0.011$ for the analytic model and $\langle p\rangle=0.009$ \pm 0.003 for the Monte Carlo simulations. Thus the theoretical predictions of $\langle p\rangle$ are in good agreement with each other and with the observational measurement. We conclude that we are able to recreate easily the observed lensing signal with a simple, physically motivated model, strengthening the case for galaxy-galaxy lensing as the cause of the observed image polarisation.

It is clear from Figure 2 that the mean image polarization of our faint galaxies decreases with limiting magnitude. For completeness, we show in Table 1 the mean image polarization of the faint galaxies as a function of limiting magnitude. From this table it apparent that $\langle p\rangle$ decreases to $r_{s} \sim 25$ and is negligible at the fainter limits. In $\S 2$ it was argued that such a trend is expected on the basis of the decrease in image size and the associated increase in the errors in the determination of the orientations of the faint galaxies.

Using the Monte Carlo simulations, we can estimate the effect of the errors associated with the determination of the image orientations. Simulations such as those outlined in $\S 3.5$ were run for our fiducial lens and source samples (i.e., $\left.20 \leq r_{d} \leq 23,23<r_{s} \leq 24\right)$, and the net image polarization of each faint galaxy was determined. In $\S 3.5, P_{\phi}(\phi)$ was calculated assuming no errors in the determination of the faint image orientations; here, we instead assign random errors to the resultant faint image orientations and investigate the effect on the inferred $\langle p\rangle$. For the sake of simplicity, we assume that the effect of all sources of noise on the determination of $P_{\phi}(\phi)$ (e.g., seeing, sky noise, pixellation, detection algorithm, etc.) can be quantified adequately in terms of one number, the error associated with the determination of the faint image position angle, which we have estimated for the observed fiducial source sample in $\S 2.2$.

To begin, we assume the error in the faint image position angle, $\phi$, is independent of the image ellipticity (which is not strictly true, as discussed in $\S 2$ ) and assign Gaussian distributed errors to each faint Monte Carlo image $\phi$ with a chosen standard deviation $\sigma_{\phi}$. In Table 4 the resultant mean polarization expected on the basis of the fiducial model is listed as a function of $\sigma_{\phi}$. It is clear that for $\sigma_{\phi} \gtrsim$ $25^{\circ}$ the measured $\langle p\rangle$ differs significantly from the true $\langle p\rangle$ and is a strongly decreasing function of $\sigma_{\phi}$.

To assess the importance of the inclusion of realistic errors in the determination of $\langle p\rangle$ in Monte Carlo simulations of the fiducial lens and source samples, another set of simulations was run in which, rather than assuming $\sigma_{\phi}$ is independent of $\epsilon$, the data from Table 2 (i.e., the observational estimates $\sigma_{\phi}$ as a function of image ellipticity) were used to 
TABLE 4

\begin{tabular}{|c|c|}
\hline \multicolumn{2}{|c|}{$\begin{array}{l}\text { DEGRADATION OF IMAGE } \\
\text { POLARIZATION DUE } \\
\text { TO ERRORS IN IMAGE } \\
\text { POSITION ANGLES }\end{array}$} \\
\hline$\sigma_{\phi}$ & $\langle p\rangle(1 \sigma$ errors $)$ \\
\hline & $0.009 \pm 0.003$ \\
\hline & $0.009 \pm 0.003$ \\
\hline $10 .$. & $0.009 \pm 0.003$ \\
\hline $15 \ldots$ & $0.008 \pm 0.003$ \\
\hline $20 .$. & $0.007 \pm 0.003$ \\
\hline $25 \ldots$ & $0.006 \pm 0.003$ \\
\hline & $0.005 \pm 0.003$ \\
\hline & $0.004 \pm 0.003$ \\
\hline & $0.003 \pm 0.003$ \\
\hline $45 \ldots \ldots \ldots \ldots$ & $0.003 \pm 0.003$ \\
\hline & $0.002 \pm 0.003$ \\
\hline
\end{tabular}

assign appropriate Gaussian-distributed errors to the position angles of the simulation images on the basis of their ellipticities. That is, images with low values of $\epsilon$ were assigned errors in $\phi$ using a broader distribution than that used to assign errors to images with larger $\epsilon$. The procedure was implemented as follows. For each faint Monte Carlo galaxy, its image ellipticity and orientation after being lensed by the foreground galaxies was calculated. Based on its ellipticity, the true position angle of the image, $\phi$, was then "corrupted" by the addition of a randomly chosen error from the appropriate distribution as given in Table 2. The function $P_{\phi}(\phi)$ was then determined using the set of corrupted image position angles, and the resultant $\langle p\rangle$ was found. For our fiducial annulus of $5^{\prime \prime} \leq \theta \leq 34^{\prime \prime}$, the inclusion of the $\phi$ errors results in a reduction of order $15 \%$ in $\langle p\rangle$ from its true value, which is well within the formal $1 \sigma$ error anticipated on the basis of the model alone. Therefore, for all remaining analysis and fitting of models to the observed image polarization, we will neglect the inclusion of errors associated with the image orientation.

Thus far, the image polarization of the faint galaxies has been investigated only in a fixed aperture of inner radius $5^{\prime \prime}$ and outer radius $34^{\prime \prime}$, centered on each of the bright galaxies. We now investigate the radial dependence of the polarization signal in order to determine the level at which the parameters of our lensing model can be constrained. The image polarization will diminish for lines of sight passing outside the individual dark matter halos of the lenses and, so, the scale at which the observed $\langle p\rangle$ approaches zero is an indication of the angular extent of the halos. In Figure $5 a$ we show $\langle p\rangle$ for our fiducial subsample of galaxies calculated in annuli of inner radius $5^{\prime \prime}$ and outer radius $\theta_{\max }$, where $15^{\prime \prime} \leq \theta_{\max } \leq 145^{\prime \prime}$. The observed points were obtained from a weighted least-squares fit of equation (3.26) to the corresponding observed functions $P_{\phi}(\phi)$, exactly as was done for the previous annulus of $5^{\prime \prime} \theta \leq 34^{\prime \prime}$. The weights were obtained by bootstrap resampling of the data to estimate the variance in each $\phi$ bin, and the error bars on Figure $5 a$ are the formal errors from the leastsquares fit. These measurements of $\langle p\rangle$ are, of course, not independent, but they do serve to illustrate that $\langle p\rangle$ is significantly nonzero when averaged over scales up to $\theta_{\max } \sim 90^{\prime \prime}$, which suggests that the dark halos of the lenses extend to large radii $\left(\gtrsim 100 h^{-1} \mathrm{kpc}\right)$. In addition, they serve to demonstrate the argument put forth in $\S 3.6$ that our choice of annulus $5^{\prime \prime} \leq \theta \leq 34^{\prime \prime}$ roughly maximizes the signal-to-noise ratio.
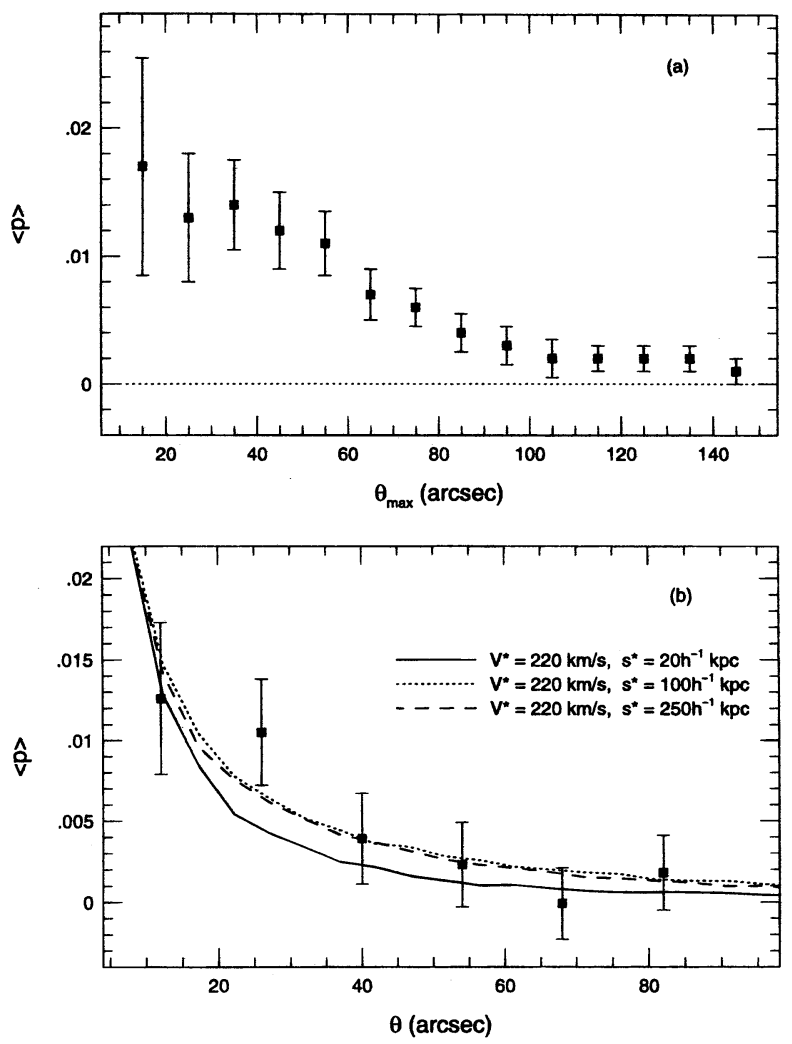

FIG. 5.-Angular variation of image polarization for foreground galaxies with $20 \leq r_{d} \leq 23$ and background galaxies with $23<r_{s} \leq 24$. (a) Variation of $\langle p\rangle$ with increasing annulus outer radius, $\theta_{\max }$. (b) Variation of $\langle p\rangle$ with differential lens-source separation, $\theta$. Theoretical estimates of $\langle p\rangle(\theta)$ for fiducial $L^{*}$ galaxy gravitational lenses (see $\S 3$ ) with different scaling radii, $s^{*}$, are also shown.

A more ambitious approach to constraining our model parameters is possible if we attempt to measure the sizes of the lens galaxies by determining the variation of polarization with the differential source-lens separation, $\theta$. Again using our fiducial subsample of galaxies, we have determined $\langle p\rangle$ for independent radial bins, $\theta$. The results are exhibited in Figure $5 b$, where, again, the error bars are the formal error obtained from the weighted least-squares fit. In addition, we have computed the mean polarization of the faint galaxy images in the Monte Carlo simulations as a function of $\theta$ for our fiducial $L^{*}$ galaxy model assuming different values of the outer radial scale, $s^{*}$. Results for $V^{*}=220 \mathrm{~km} \mathrm{~s}^{-1}$ and $s^{*}=20 h^{-1} \mathrm{kpc}, 100 h^{-1} \mathrm{kpc}, 250$ $h^{-1} \mathrm{kpc}$ are shown in Figure $5 b$.

Using the observed variation of $\langle p\rangle$ with lens-source separation from Figure $5 b$ we now attempt to constrain the model parameters $V^{*}$ and $s^{*}$. To begin, we fix one of $s^{*}$ or $V^{*}$ and vary the other parameter. The variation of $\langle p\rangle$ with $\theta$ is then computed for each of the Monte Carlo models and compared to the data in Figure $5 b$ using a $\chi^{2}$ statistic. Formal best-fit values of the parameters are those for which $\chi^{2}$ is a minimum. Since $\langle p\rangle$ for our fiducial annulus of $5^{\prime \prime} \leq \theta \leq 34^{\prime \prime}$ was well fitted by $V^{*}=220 \mathrm{~km} \mathrm{~s}^{-1}$ and $s^{*}=100 h^{-1} \mathrm{kpc}$, we will use these as the fixed parameter values.

Keeping $V^{*}$ constant at $220 \mathrm{~km} \mathrm{~s}^{-1}, s^{*}$ was increased incrementally from $5 h^{-1} \mathrm{kpc}$ to $200 h^{-1} \mathrm{kpc}$ in the simulations and the variation of $\langle p\rangle$ with $\theta$ was determined for each value of $s^{*}$. Shown in Figure $6 a$ is the reduced $\chi^{2}$ (i.e., 

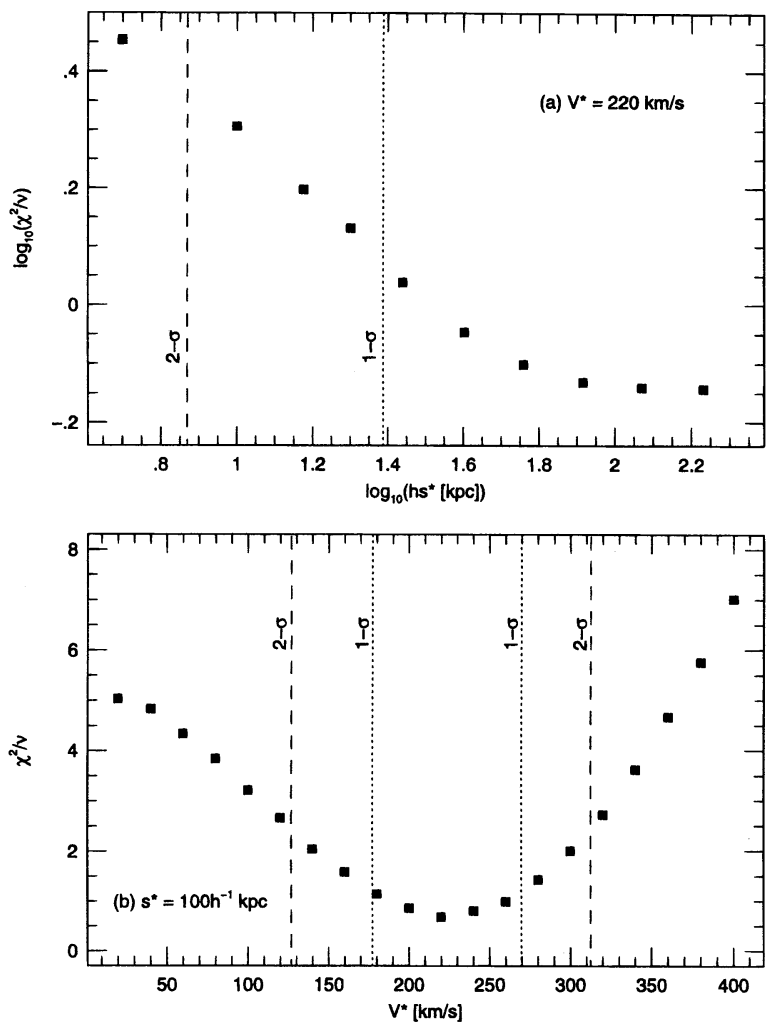

Fig. 6. - $\chi^{2}$ per degree of freedom obtained from comparison of Monte Carlo $\langle p\rangle(\theta)$ to the data in Fig. 5b. Formal 1 and $2 \sigma$ confidence bounds are indicated by the vertical dotted and dashed lines, respectively. $(a)$ Dependence of $\chi^{2} / v$ on $s^{*}$, where $V^{*}=220 \mathrm{~km} \mathrm{~s}^{-1}$. (b) Dependence of $\chi^{2} / v$ on $V^{*}$, where $s^{*}=100 h^{-1} \mathrm{kpc}$.

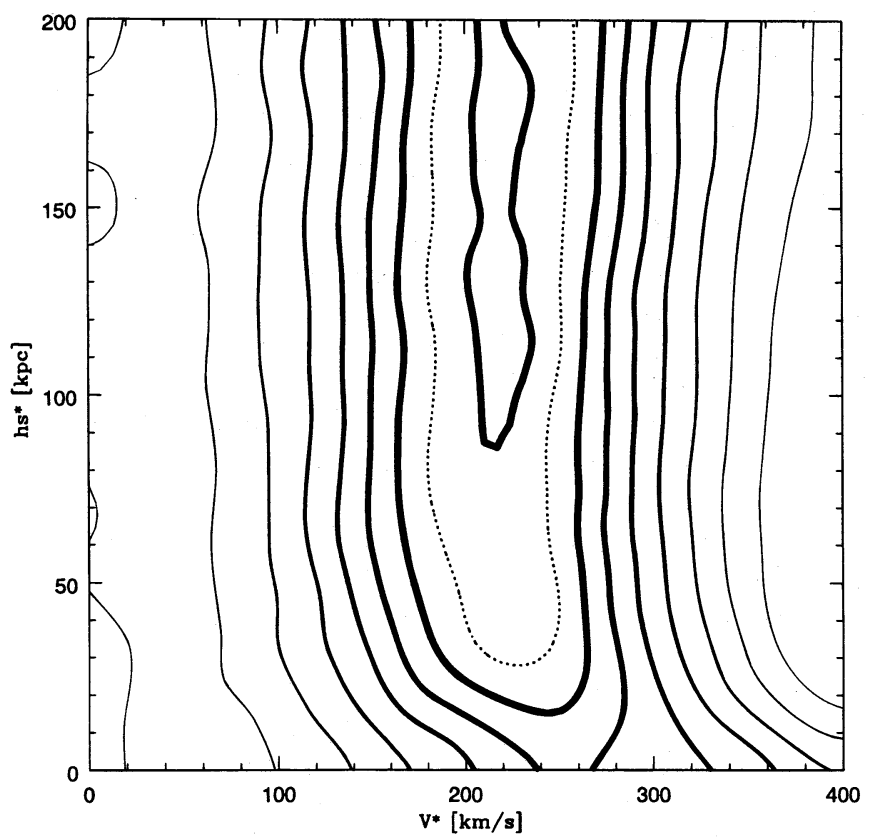

Fig. 7.-Logarithmically spaced contours of constant $\chi^{2}$ per degree of freedom obtained from a comparison of the Monte Carlo $\langle p\rangle(\theta)$ to the data in Fig. $5 b$, where the parameters $V^{*}$ and $s^{*}$ were varied simultaneously. The contours range from $\chi^{2} / v=8$ (thin line, edges of figure) to $\chi^{2} / v=0.8$ (thick line, center of figure). The dotted line indicates the contour for which the $\chi^{2}$ per degree of freedom is unity. A formal minimum in $\chi^{2} / v$ is reached at $V^{*} \sim 220 \mathrm{~km} \mathrm{~s}^{-1}$ for $s^{*} \gtrsim 100 h^{-1} \mathrm{kpc}$.
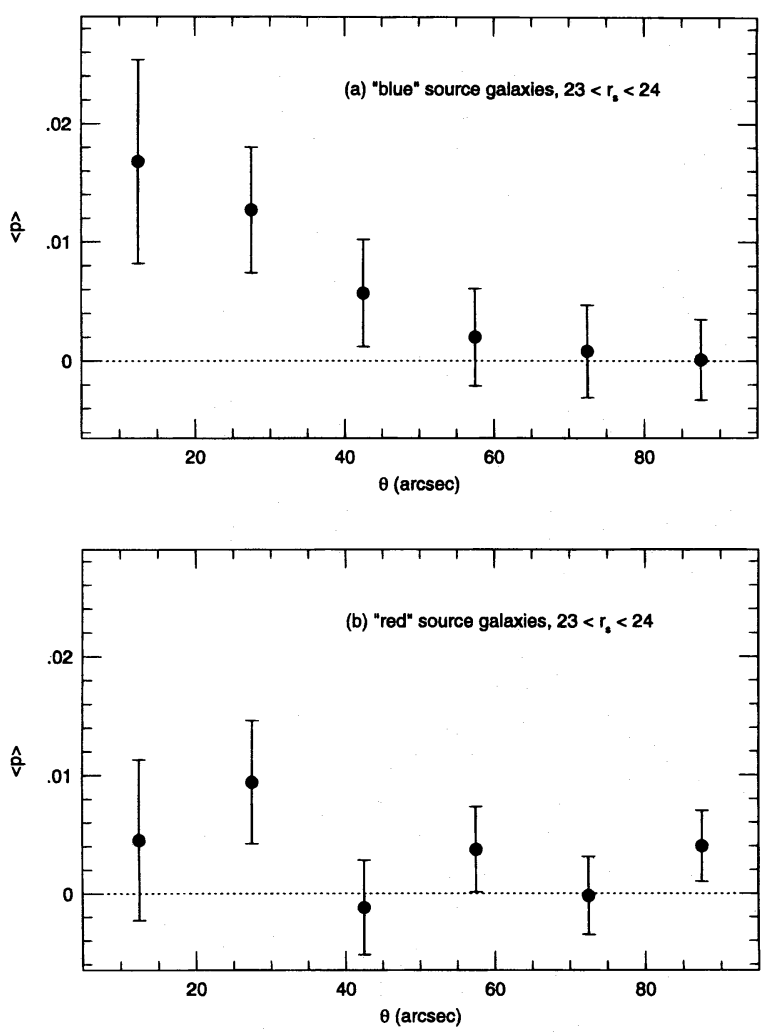

Fig. 8.-Angular variation of image polarization for the (a) "blue," $(g-r)<0.53$, and $(b)$ "red," $(g-r)>0.53$, source subsamples as a function of differential lens-source separation, $\theta$, where $23<r_{s} \leq 24$.

$\chi^{2}$ per degree of freedom) obtained from the comparison of the model $\langle p\rangle(\theta)$ with the data in Figure $5 b$. From this figure, then, the reduced $\chi^{2}$ decreases monotonically from large values at small $s^{*}$ to a constant small value at large $s^{*}$ $\left(\gtrsim 100 h^{-1} \mathrm{kpc}\right)$. That is, for values of $s^{*} \gtrsim 100 h^{-1} \mathrm{kpc}$, the expected variation of $\langle p\rangle$ with $\theta$ is essentially constant, as discussed in $\S$ 3.6. Therefore, we are unable to determine a unique best-fit value of $s^{*}$. The formal $\chi^{2}$ minimization suggests that the halos of the lenses are large, $s^{*} \gtrsim 100 h^{-1}$ $\mathrm{kpc}$; however, $s^{*} \sim 25 h^{-1} \mathrm{kpc}$ can only be ruled out at the $1 \sigma$ level. Very small halos $\left(s^{*} \sim 10 h^{-1} \mathrm{kpc}\right)$ are excluded at the $2 \sigma$ level. The formal 1 and $2 \sigma$ confidence levels on $s^{*}$ are indicated in Figure $6 a$ by the vertical dotted and dashed lines, respectively.

Next, we hold $s^{*}$ constant at $100 h^{-1} \mathrm{kpc}$ in the simulations and vary $V^{*}$. The values of the reduced $\chi^{2}$ obtained by comparing the model $\langle p\rangle(\theta)$ with the observations in Figure $5 b$ are shown in Figure $6 b$. It is clear from this figure that the reduced $\chi^{2}$ is minimized for $V^{*} \simeq 220 \mathrm{~km} \mathrm{~s}^{-1}$. Unlike $s^{*}$, it is possible to obtain both lower and upper 1 and $2 \sigma$ confidence bounds on $V^{*}$, and these are indicated in Figure $6 b$ by the vertical dotted and dashed lines, respectively. From this figure, then, the formal $90 \%$ confidence limits on $V^{*}$ are $\sim 140 \mathrm{~km} \mathrm{~s}^{-1}$ and $\sim 300 \mathrm{~km} \mathrm{~s}^{-1}$.

Figure 7 is a contour representation of the the reduced $\chi^{2}$ obtained when the Monte Carlo $\langle p\rangle(\theta)$ is compared to the data in Figure $5 b$ and the parameters $V^{*}$ and $s^{*}$ are varied simultaneously. The lines are contours of constant $\chi^{2}$ per degree of freedom, logarithmically spaced from $\chi^{2} / v=8$ (edges of the plot) to $\chi^{2} / v=0.8$ (center of the plot). The width of the contour lines is indicative of the value of the 
reduced $\chi^{2}$ with the widest lines corresponding to the smallest values and vice versa. The contour corresponding to a $\chi^{2}$ per degree of freedom of unity is indicated by the dotted line. As in Figure 6, there is a clear minimization of the reduced $\chi^{2}$ in the region of $V^{*} \sim 220 \mathrm{~km} \mathrm{~s}^{-1}$, resulting in a formal "best-fit" value for this parameter, but such is not the case for $s^{*}$.

Finally, it is clear from equation (3.17) that the redshift distributions of our fiducial subsamples of lenses $\left(20 \leq r_{d} \leq\right.$ $23)$ and sources $\left(23<r_{s} \leq 24\right)$ will have some overlap. We expect the measured mean polarization to be greatest for the most distant sources and for the case that all the sources are at higher redshifts than the candidate lenses. We are therefore driven to look at using additional information to separate better the foreground lenses from the background sources. One obvious possibility is to use the colors of the faint sources from our matched $g$-and $r$-band data. We thus split the source sample with $23<r_{s} \leq 24$ into a "red" half $[(g-r)>0.53]$ and a "blue" half $[(g-r)<0.53]$ on the basis of the $(g-r)$ colors and compute $\langle p\rangle$ for the two subsamples. Again using $5^{\prime \prime} \leq \theta \leq 34^{\prime \prime}$, we find $\langle p\rangle_{\text {blue }}=$ $0.016 \pm 0.008$ and $\langle p\rangle_{\text {red }}=0.008 \pm 0.008(95 \%$ confidence limits). In addition, we compute $\langle p\rangle$ for the red and blue subsamples as in Figure $5 b$, and in Figure 8 we show the measured variation of $\langle p\rangle$ with differential lens-source separation, $\theta$. From this figure $\langle p\rangle_{\text {red }}$ appears consistent with zero over all scales, while $\langle p\rangle_{\text {blue }}$ is significantly nonzero for $\theta \leq 30^{\prime \prime}$ and there is evidence of a monotonic decrease to zero with increasing $\theta$. While not highly significant, these data suggest a higher mean polarization of the blue images than the red images, a result that makes sense if a larger proportion of distant sources are blue, star-forming systems.

\section{DISCUSSION}

In this paper we have attempted to measure the induced polarization of images of distant galaxies due to weak gravitational lensing by more nearby galaxies and find a significant detection of the polarization $\langle p\rangle=0.011 \pm 0.006$ ( $95 \%$ confidence bounds), averaged over faint-bright galaxy separations of $5^{\prime \prime} \leq \theta \leq 34^{\prime \prime}$. The significance of the detection is due ot the choice of annulus over which the faint image orientation is averaged, resulting in an optimization of the signal-to-noise ratio for the detection of lensing by extended galaxy halos. We cannot explain this signal through systematic effects within our data set, and thus we suggest that it is cosmological in origin. In addition, we have presented a fiducial model that is capable of reproducing the observed gravitational lensing signal through both analytic and Monte Carlo simulations. The simulation results are quite robust to most variations of our adopted parameters, being sensitive mostly to the scaling circular velocity, $V^{*}$, and the redshift distributions. With lower significance we can claim to have measured the decrease in the gravitational lensing signal with increasing galaxy separation, consistent with a typical galaxy halo size $\gtrsim 100 h^{-1}$ $\mathrm{kpc}$, though very small halos $\left(\sim 10 h^{-1} \mathrm{kpc}\right)$ are excluded at only the $2 \sigma$ level.

The best-fitting parameters for our fiducial model as derived from the Monte Carlo simulations can be used to estimate the masses of the lens galaxies contained within a radius, $r$. For $V^{*}=220 \mathrm{~km} \mathrm{~s}^{-1}$ and $s^{*}=100 h^{-1} \mathrm{kpc}$ and using the $90 \%$ confidence limits on $V^{*}$ derived above, we find $M\left(100 h^{-1} \mathrm{kpc}\right) \sim 9_{-5}^{+7} \times 10^{11} h^{-1} M_{\odot}$. Letting $s^{*} \rightarrow \infty$, we obtain a maximum contained mass of $M_{\max }(100$ $\left.h^{-1} \mathrm{kpc}\right) \sim 1.1_{-0.6}^{+1.0} \times 10^{12} h^{-1} M_{\odot}$ for our allowed range of $V^{*}$. From our model calculations, then, we estimate an allowed range for the masses of the lens halos to be $M(100$ $\left.h^{-1} \mathrm{kpc}\right) \sim 1.0_{-0.5}^{+1.2} \times 10^{12} h^{-1} M_{\odot}$.

Given our fiducial median redshift, the typical luminosities of the lens galaxies in our sample are $L_{V} \sim 5 \times 10^{9}$ $h^{-2} L_{\odot}$. We thus estimate rest-frame mass-to-light ratios inside a radius of $100 h^{-1} \mathrm{kpc}$ of our lens galaxies to be $M / L_{V} \sim 200_{-120}^{+220} h\left(M / L_{V}\right)_{\odot}$ for $\Omega_{0}=1$. Recent results from Carlberg et al. (1995) indicate the required mass-to-light ratio for closure density is $M / L_{V}=(1630 \pm 130) h\left(M / L_{V}\right)_{\odot}$, and we therefore estimate the fraction of the closure density contained in the central regions of galaxies to be $\Omega_{g} \sim$ $0.2_{-0.1}^{+0.2}$.

Few measurements exist for galaxy masses on the scales probed here. Perhaps the best existing estimates of the extent and masses of galaxy halos come from statistical studies of satellite galaxies. Zaritsky \& White (1994) have analyzed a sample of companion galaxies to isolated local spirals and find that the typical masses out to $150 h^{-1} \mathrm{kpc}$ are in the range $1-2 \times 10^{12} h^{-1} M_{\odot}$, consistent with our findings: $M\left(150 h^{-1} \mathrm{kpc}\right) \sim 1.5_{-1.0}^{+1.6} \times 10^{12} h^{-1} M_{\odot}$. Since the assumptions underlying the two techniques are very different, the close agreement between the two methods is encouraging.

We conclude that weak gravitational lensing is a viable and potentially powerful probe of the outer parts of normal galaxies-regions that are inaccessible to strong lensing studies (Breimer \& Sanders 1993). That our polarization measurement has been achieved using only a single CCD frame under conditions of modest seeing augurs well for future investigations. When planning such an observation, a compromise has to be reached between area coverage and depth when accruing the source and lens samples. Galaxy counts increase at a rate of about two per magnitude; hence, the number of galaxy-lens pairs should quadruple for each magnitude, and, theoretically, the random error in the polarization measurement should halve, making it advantageous to go fainter. In practice, the limiting magnitude for such an investigation is set by our ability to assign accurate shapes to individual source galaxies, and we have seen that the empirical limit for our data is $r \sim 24$ (Fig. 2). Note that in the absence of scale evolution, beyond a redshift $z \sim 0.5$ the image sizes of galaxies are relatively fixed (although see Smail et al. 1995), and to increase the depth of the sample by $\sim 1$ mag takes $\sim 5$ times as much integration. Consequently, even when the galaxy images can be measured accurately, galaxy-lens pairs are accumulated at about the same rate by taking additional CCD frames as by increasing the depth of exposure of an individual field.

It is clear from our observations and simulations that atmospheric seeing seriously degrades the image polarization at faint magnitudes where the signal-to-noise ratio would otherwise increase. This should not be a problem for deep HST images, and it is important to repeat this measurement using the deepest WFPC2 fields as they become available. Using such data, we anticipate being able to measure image ellipticities accurately to $r=26$. In this case, the lensing signal should go up by a factor of 3 , while the noise should go down by a factor of 4 for a given area. Currently, a sample of $\sim 10$ WFPC2 fields would be appropriate for our analysis, so that the signal-to-noise ratio may improve by a factor of 30 (although this will depend upon 
the galaxy redshift distribution). Therefore, it should be possible to improve the accuracy of the measurement substantially and constrain both the redshift distribution of faint galaxies and the sizes of their halos.

The eventual limitations to this technique are probably connected with our incomplete knowledge of the galaxy redshift distribution and the unknown contribution to the statistical signal of a minority of unusual lenses and tidal interactions. Despite these concerns, the prospect of studying galaxies on a scale in which their dynamical times are comparable to their ages is exciting. In this regime we can observe the infall of the outer parts of the galaxies and thus, in some sense, see the galaxies assembling. This encourages us to devote more observational effort to measuring galaxyinduced polarization.

We are indebted to Jeremy Mould and Todd Small for acquiring the observations used for this analysis and to them, David Hogg, Gordon Squires, and Tony Tyson for helpful suggestions. Support under NSF contract AST 9223370, the NASA HPCC program at Los Alamos National Laboratory (T. G. B.), and a NATO Advanced Fellowship (I. R. S.) is gratefully acknowledged.

\section{APPENDIX A}

\section{PARTICLE DISTRIBUTION FUNCTION ASSOCIATED WITH MODEL POTENTIAL}

In $\S 3.1$, we introduced a simple galaxy model with a density distribution given by equation (3.1). Associated with this density distribution is the three-dimensional potential

$$
\phi(r)=-\int_{r}^{\infty} d r^{\prime} \frac{G M\left(r^{\prime}\right)}{r^{\prime 2}}=-V_{c}^{2}\left[\frac{\tan ^{-1} x}{x}+\frac{1}{2} \ln \left(1+x^{-2}\right)\right],
$$

where $x=r / s$. In order to demonstrate that this potential is physically realizible, we compute the associated isotropic distribution function for dark matter particles assuming that they are all of the same mass $m$ and ignoring the luminous stars, which ought only to contribute at small radius. We denote this distribution function by $f(E)$, where $E=v^{2} / 2+\phi$ is the specific energy. If we now regard $\rho$ as a function of $\phi$, then we can write

$$
\rho(\phi)=42^{1 / 2} \pi m \int_{\phi}^{0} d E(E-\phi)^{1 / 2} f(E),
$$

(see, e.g., Binney \& Tremaine 1987). This integral equation is easily cast in Abel form and can be solved to give

$$
f(E)=\frac{1}{2^{1 / 2} \pi^{2} m} \int_{E}^{0} d \phi(\phi-E)^{1 / 2} \frac{d^{3} \rho}{d \phi^{3}},
$$

where we have imposed the boundary condition that $\rho$ and its derivatives $\rightarrow 0$ as $\phi \rightarrow 0$. This distribution function can be evaluated numerically and is found to decline monotonically with $E$. In addition, $d^{3} \rho / d \phi^{3}<0$. These properties suffice to ensure stability to small perturbations (see, e.g., Binney \& Tremaine 1987).

\section{APPENDIX B}

\section{INFLUENCE OF WEAK LENSING ON GALAXY AUTOCORRELATION FUNCTION}

Curiously, weak gravitational lensing produces an observable effect on the autocorrelation function of distant galaxies. It might be thought that the magnification of more distant galaxies by intervening lenses would result in a positive contribution to the measured autocorrelation function. However, the deflection of the galaxy images also expands the separation between galaxies, and this turns out to be the larger effect. If the slope of the galaxy counts can be expressed as

$$
\frac{d \log N}{d r}=q,
$$

where $q \sim 0.3$, then the surface density of background galaxies at a distance $\theta$ from a given lens galaxy will be given be enhanced by a factor $1+w^{\prime}(\theta)$, where

$$
w^{\prime}(\theta) \simeq(2.5 q-1) \mu(\theta),
$$

and $\mu(\theta)$ is the magnification (see, e.g., Narayan 1989). The contribution of gravitational lensing to the correlation function turns out to be negative, reducing the very small scale clustering since the distance between galaxies has been increased. We 
can relate $w^{\prime}(\theta)$ to the polarization, $p(\theta)$, using equations (3.2) and (3.7), and we find

$$
\frac{w^{\prime}(\theta)}{p(\theta)}=(2.5 q-1)\left[\frac{1-\left(1+X^{-2}\right)^{-1 / 2}}{X G(X)}\right] \text {. }
$$

This increases from $\sim-1 / 4$ for $X \ll 1$ to $\sim-1 / 16 X$ for $X \gg 1$. This ratio is always small and so cannot affect the measured image polarization. However, it can have an effect upon the autocorrelation function of galaxies fainter than those scrutinized $-!$ here by canceling a significant fraction of the true correlation on scales $\$ 10^{\prime \prime}$. On scales of $\sim 15^{\prime \prime}-20^{\prime \prime}$, however, the effect is very small, and it is negligible on scales $\gtrsim 50$ ". Therefore, weak gravitational lensing cannot account for the observed low clustering amplitude of very faint galaxies on scales $\gtrsim 15^{\prime \prime}$ (see, e.g., Brainerd et al. 1995; Efstathiou et al. 1991).

\section{REFERENCES}

Aaronson, M., \& Mould, J. R. 1983, ApJ, 265, 1

Binney, J. J., \& Tremaine, S. D. 1987, Galactic Dynamics (Princeton: Princeton Univ. Press)

Brainerd, T. G., Smail, I., \& Mould, J. R. 1995, MNRAS, 275, 781

Blandford, R. D., \& Narayan, R. 1992, ARA\&A, 30, 311

Breimer, T. G., \& Sanders, R. H. 1993, A\&A, 274, 96

Carlberg, R., Yee, H. K., Ellingson, E., Abraham, R., Gravel, P., S., \& Pritchet, C. J. 1995, preprint astro-ph/9509034

de Zeeuw, T., \& Franx, M. 1991, ARA\&A, 29, 239

Dressler, A., et al. 1996, in preparation

Efstathiou, G., Bernstein, G., Katz, N., Tyson, J. A., \& Guhathakurta, P. 1991, ApJ, 380, L47

Fabbiano, G. 1989, ARA\&A, 27, 87

Fich, M., \& Tremaine, S. D. 1991, ARA\&A, 29, 409

Fort, B., \& Mellier, Y. 1994, Astron. Astrophys. Rev., 5, 239

Kovner, I., \& Milgrom, M. 1987, ApJ, 321, L113

Kristian, J. 1967, ApJ, 147, 864

Kristian, J., \& Sachs, R. K. 1966, ApJ, 143, 379
Lilly, S. 1993, ApJ, 411, 501

Loveday, J., Peterson, B. A., Efstathiou, G., Maddox, S. J., \& Sutherland, W. J. 1992 ApJ, 390338

Mould, J., Blandford, R., Villumsen, J., Brainerd, T., Smail, I., Small, T., \& Kells, W. 1994, MNRAS, 271, 31 (Paper I)

Narayan, R. 1989, ApJ, 339, L53

Phillips, S. 1985, Nature, 314, 721

Schneider, P., Ehlers, J., \& Falco, E. 1992, Gravitational Lenses (Berlin: Springer)

Smail, I., Hogg, D. W., Yan, L., \& Cohen, J. G. 1995, ApJ, 449, L105

Tresse, L., Hammer, F., LeFevre, O., \& Proust, D. 1993, A\&A, 277, 53

Tyson, J. A. 1985, Nature, 316, 799

Tyson, J. A., Valdes, F., Jarvis, J. F., \& Mills, A. P. 1984, ApJ, 281, L59

Valdes, F. 1982, FOCAS Manual, NOAO

Valdes, F., Tyson, J. A., \& Jarvis, J. F. 1983, ApJ, 271, 431

Webster, R. L. 1983, Ph.D. thesis, Univ. of Cambridge

Zaritsky, D., \& White, S. D. M. 1994, ApJ, 435, 599 